\title{
Griffin: Framework for clinical cancer subtyping from nucleosome profiling of cell-free DNA
}

Anna-Lisa Doebley ${ }^{1,2,3}$, Minjeong Koํ․ Hanna Liao ${ }^{2,4}$, A. Eden Cruikshank ${ }^{2}$, Caroline Kikawa ${ }^{3}$, Katheryn Santos ${ }^{5}$, Joseph Hiatt ${ }^{1}$, Robert D. Patton ${ }^{1}$, Navonil De Sarkar ${ }^{1}$, Anna C.H. Hoge ${ }^{1}$, Katharine Chen ${ }^{2}$, Zachary T. Weber ${ }^{6}$, Mohamed Adil ${ }^{1,7}$, Jonathan Reichel ${ }^{7}$, Paz Polak ${ }^{8}$, Viktor A. Adalsteinsson ${ }^{9}$, Peter S. Nelson ${ }^{1}$, Heather A. Parsons ${ }^{5}$, Daniel G. Stover ${ }^{6}$, David MacPherson ${ }^{1,4}$, Gavin $\mathrm{Ha}^{1,4+}$

${ }^{1}$ Divisions of Public Health Sciences and Human Biology, Fred Hutchinson Cancer Research Center, 1100 Fairview Ave. N, Seattle, WA 98109

${ }^{2}$ Molecular and Cellular Biology Graduate Program, University of Washington, 1959 NE Pacific St, Seattle WA 98195

${ }^{3}$ Medical Scientist Training Program, University of Washington, 1959 NE Pacific St, Seattle, WA 98195

${ }^{4}$ Department of Genome Sciences, University of Washington, 1959 NE Pacific St, Seattle, WA 98195

${ }^{5}$ Dana-Farber Cancer Institute, 450 Brookline Ave, Boston, MA 02215

${ }^{6}$ The Ohio State University Comprehensive Cancer Center, 460 W. $10^{\text {th }}$ Ave, Columbus, OH 43210

${ }^{7}$ Laboratory Medicine and Pathology, University of Washington, 1959 NE Pacific St, Seattle, WA 98195

${ }^{8}$ Icahn School of Medicine, Mount Sinai, One Gustave L. Levy Place, New York, NY 10029

${ }^{9}$ Broad Institute of MIT and Harvard, 415 Main Street, Cambridge, MA 02142

${ }^{\dagger}$ Correspondence: Gavin Ha (gha@fredhutch.org)

NOTE: This preprint reports new research that has not been certified by peer review and should not be used to guide clinical practice. 
medRxiv preprint doi: https://doi.org/10.1101/2021.08.31.21262867; this version posted September 3, 2021. The copyright holder for this preprint (which was not certified by peer review) is the author/funder, who has granted medRxiv a license to display the preprint in perpetuity.

It is made available under a CC-BY-NC-ND 4.0 International license.

\section{Abstract (150 words)}

2 Cell-free DNA (cfDNA) has the potential to inform tumor subtype classification and help guide

3 clinical precision oncology. Here we developed Griffin, a new method for profiling nucleosome

4 protection and accessibility from cfDNA to study the phenotype of tumors using as low as $0.1 \mathrm{x}$

5 coverage whole genome sequencing (WGS) data. Griffin employs a novel GC correction

6 procedure tailored to variable cfDNA fragment sizes, which improves the prediction of chromatin

7 accessibility. Griffin achieved excellent performance for detecting tumor cfDNA in early-stage

8 cancer patients $(A \cup C=0.96)$. Next, we applied Griffin for the first demonstration of estrogen

9 receptor (ER) subtyping in metastatic breast cancer from cfDNA. We analyzed 254 samples from

10139 patients and predicted ER subtype with high performance (AUC=0.89), leading to insights

11 about tumor heterogeneity. In summary, Griffin is a framework for accurate clinical subtyping and

12 can be generalizable to other cancer types for precision oncology applications. 


\section{Introduction}

14 Accurate cancer diagnosis and subtype classification are critical for guiding clinical care and

15 precision oncology. Current approaches to determine tumor subtype require a tissue biopsy,

16 which is often difficult to obtain from patients with metastatic cancer. Therefore, at the time of

17 recurrence or metastatic cancer diagnosis, treatment options may often be informed by clinical

18 diagnostics from the primary tumor. However, molecular changes in the tumor can emerge during

19 metastatic progression and in the context of therapeutic resistance. Moreover, surveying

20 molecular changes is challenging because repeated biopsies are problematic and not routine in

21 clinical practice for solid tumors.

23 Cell-free DNA (cfDNA) is DNA released into circulation by cells during apoptosis and necrosis. ${ }^{1}$

24 In patients with cancer, a portion of this cfDNA is released from tumor cells, called circulating

25 tumor DNA (ctDNA). The analysis of ctDNA can address the challenges in tissue accessibility and

26 has demonstrated great potential for clinical utility. ${ }^{2-9}$ Much of the current research and clinical

27 efforts have focused on the detection of genetic alterations in ctDNA. Shallow coverage

28 sequencing of cfDNA, including ultra-low pass whole genome sequencing (ULP-WGS, 0.1x),

29 provides a cost-effective and scalable solution for estimating the tumor fraction (fraction of the

30 cfDNA that is tumor derived) from the analysis of genomic copy number alterations. ${ }^{10-13}$

31 Sequencing analysis of genomic alterations from ctDNA have helped to distinguish molecular

32 subsets of tumors. ${ }^{14,15}$ However, these genomic alterations, including somatic mutations, may not

33 always fully explain treatment failure or identify therapeutic targets, exemplifying a major limitation

34 of cancer precision medicine.

36 Tumor subtypes are often characterized by distinct transcriptional regulation, which can change

37 during treatment resistance, leading to different clinical tumor phenotypes. For example, prostate

38 and lung cancers may undergo trans-differentiation from adenocarcinoma to small-cell 
neuroendocrine phenotypes. ${ }^{16-20}$ For metastatic breast cancer (MBC), treatment is guided based on clinical subtypes determined by the expression of the estrogen receptor (ER), progesterone

41 receptor (PR), and human epidermal growth factor receptor 2 (HER2), often in the primary

42 tumor ${ }^{21}$; endocrine therapies are prescribed to patients with ER-positive (ER+) or PR-positive

$43(\mathrm{PR}+)$ carcinomas while patients with HER2 positive tumors are prescribed anti-HER2 drugs.

44 Patients with tumors absent for expression of all three receptors have triple negative breast

45 cancer (TNBC) and receive chemotherapy. ${ }^{22}$ However, receptor conversions during primary and metastatic disease progression have been frequently observed, including $\sim 20 \%$ of patient tumors

47 switching from ER+ to ER-negative (ER-) subtypes. ${ }^{23-28}$ Furthermore, similar to the presence of 48 intra-tumor genomic heterogeneity in breast cancer, mixtures of clinical subtypes may also co49 exist across or within metastatic lesions in the same patient, presenting major clinical 50 challenges. ${ }^{29,30}$ Therefore, accurate subtype classification and identification of transcriptional 51 patterns underlying emergent clinical phenotype during therapy has critical implications for 52 studying mechanisms of resistance and informing treatment decisions.

54 Recent studies have shown that the computational analysis of cfDNA fragmentation patterns from 55 genome sequencing data can reveal the occupancy of nucleosomes in cells-of-origin. ${ }^{31-36}$ When 56 DNA is released into the peripheral blood following cell death, they are protected from degradation 57 by nucleosomes. ${ }^{1}$ At accessible genomic locations, such as at actively bound transcription factor 58 binding sites (TFBSs) and open chromatin regions, nucleosomes are positioned in an organized 59 manner that allows access for DNA binding proteins ${ }^{37}$ (Fig. 1a). This nucleosome organization 60 results in a loss of sequencing coverage, reflecting DNA degradation at the unprotected binding 61 site with peaks of coverage at the surrounding protected locations.

63 Applications of nucleosome profiling from cfDNA have been demonstrated for cancer detection 64 and tumor tissue-of-origin prediction, including the analysis of shorter cfDNA fragments which 
65 tend to be enriched from tumor cells. ${ }^{38-41}$ While tumor subtyping from cfDNA has been explored

66 in prostate cancer by analyzing TFBS locations ${ }^{42}$, to our knowledge there have not been

67 demonstrations of subtype classification from cfDNA in other cancers. Specifically, predicting

68 histological subtypes in breast cancer has not been shown from cfDNA. Furthermore, current

69 cfDNA nucleosome profiling approaches have not been optimized for ULP-WGS data. Studying

70 the clinical phenotype of tumors from ctDNA remains challenging due to lack of robust

71 computational methods but has obvious potential clinical benefits for guiding treatment decisions

72 in patients with metastatic cancer.

74 In this present study, we developed a computational framework called Griffin to classify tumor

75 subtypes from nucleosome profiling of cfDNA. Griffin overcomes current analytical challenges to

76 profiles the nucleosome accessibility and transcriptional regulation from the analysis of standard

77 cfDNA genome sequencing, including ULP-WGS (0.1x) coverage. Griffin employs a novel GC

78 correction procedure that is specific for DNA fragment sizes and therefore unique for cfDNA

79 sequencing data. We applied Griffin to perform cancer detection and tumor tissue-of-origin

80 analysis with high performance. Then, we demonstrate the first application of breast cancer ER

81 subtyping from cfDNA, showing strong classification accuracy and insights into tumor

82 heterogeneity and prognosis, all achieved from analysis of ULP-WGS data. Overall, Griffin is a

83 generalizable framework that can detect molecular changes in transcriptional regulation and

84 chromatin accessibility from cfDNA and possibly direct personalized treatment to improve patient

85 outcomes.

\section{Results}

88 Griffin framework for nucleosome profiling to predict tumor phenotype

89 We developed Griffin as an analysis framework with a new GC correction procedure to accurately

90 profile nucleosome occupancy from cfDNA. Griffin processes fragment coverage to distinguish 
91 accessible and inaccessible features of nucleosome protection (Fig. 1a). Griffin is designed to be

92 applied to whole genome sequencing (WGS) data of cfDNA from patients with cancer to quantify

93 nucleosome protection around sites of interest and is optimized to work for ULP-WGS data (Fig.

94 1b). Sites of interest can be selected from various chromatin-based assays, such as from assay

95 for transposase-accessible chromatin using sequencing (ATAC-seq) and are tailored to address

96 specific problems including cancer detection and tumor subtyping.

98 The analysis workflow begins with computing the genome-wide fragment-based GC bias for each 99 sample. Then, for the region at each site of interest, the fragment midpoint coverage is computed and reweighted to remove GC biases (Methods). Midpoint coverage rather than full fragment coverage is used because it produces higher amplitude nucleosome protection signals

102 (Supplementary Fig. 1). Next, a composite coverage profile is computed as the mean of the GCcorrected coverage across the set of sites specific for a tissue type, tumor type, transcription factor (TF), or any phenotypic comparison of interest. By examining these coverage profiles that distinguish a site as accessible and inaccessible: (a) the coverage in the window between -

107 I+ 30 bp ('central coverage'), where lower values represent increased accessibility, (b) the coverage in a window between -/+ 1000 bp ('mean coverage'), and (c) the overall nucleosome peak amplitude calculated using Fast Fourier transform (FFT, 'amplitude'). These features can be used to quantify transcription factor activity or chromatin accessibility and be used as features for

111 detection of cancer, tumor subtyping, or studying other phenotypes of interest.

\section{Griffin reduces GC biases enabling detection of tissue specific accessibility}

114 A novel aspect of Griffin is the implementation of a fragment-based GC bias correction. At open 115 chromatin regions, especially at TFBS, GC-content is non-uniform, which leads to GC-related 116 coverage biases (Fig. 2a). ${ }^{43}$ GC bias varies between samples and between different fragment 
117 lengths within a sample ${ }^{44}$ (Fig. 2b), which can have a major impact on nucleosome accessibility

118 prediction (Fig. 2c). To correct for this GC bias, for each sample and each fragment length, Griffin

119 computes the global estimated mean fragment coverage ("expected") using a fragment length

120 position model ${ }^{44}$ (Methods, Fig. 2b). Then, when calculating coverage around sites of interest,

121 each fragment is assigned a weight based on the global expected coverage. This correction

122 eliminates unexpected increases (or decreases) in coverage at binding sites, removing technical

123 biases to enhance the epithelial tissue-associated accessibility signals when analyzing WGS (9-

124 25x, Fig. 2c) cancer patient cfDNA and ULP-WGS (0.1-0.3x, Fig. 2d).

125

126 To test the performance of nucleosome profiling following Griffin GC-bias correction, we 127 compared the estimated TFBS accessibility with the amount of tumor-derived DNA (i.e. tumor 128 fraction) predicted by ichorCNA for ULP-WGS data from $191 \mathrm{MBC}$ cfDNA samples with $\geq 0.1$ tumor fraction. ${ }^{10}$ We expect the tumor fraction to be negatively corrected with the central coverage around tumor-specific sites, and positively correlated for blood-specific sites. For a blood specific

$131 \mathrm{TF}, \mathrm{LYL1}$, we observed that the central coverage at TFBSs was positively correlated with tumor 132 fraction before GC correction (Pearson's $r=0.31$ ) as expected, but this correlation was much 133 stronger after GC correction (Pearson's r=0.63, Fig. 2e). For a tumor-specific TF, GRHL2, we 134 observed a negative correlation between the central coverage and tumor fraction, as expected 135 (Pearson's r=-0.63, Supplementary Fig. 2). The mean coverage and amplitude features are also 136 correlated to tumor fraction but appeared to be less influenced by GC bias (Supplementary Fig. 137 2, Supplementary Data 1). Similar correlations between nucleosome profile features and tumor 138 fraction following GC correction were also observed for blood and cancer specific DNase I 139 hypersensitivity sites (DHSs) (Supplementary Fig. 2).

141 To quantify how GC correction reduces signal variability between samples, we examined the 142 central coverage in the 191 MBC cfDNA ULP-WGS samples for 338 TFs in the Gene Transcription 
143 Regulation Database (GTRD). ${ }^{42,45}$ For each factor, we compared the variability between the

144 central coverage and tumor fraction using the root mean squared error (RMSE) from a linear

145 regression fit before and after GC correction. For LYL1, the RMSE decreased (0.067 to 0.041),

146 indicating less inter-sample variation in the data after GC correction (Fig. 2e). Similarly, for 325

147 (96.1\%) TFs, the RMSE was decreased after GC correction, indicating reduced inter-sample

148 variability after accounting for the correlation between tumor fraction and central coverage (two-

149 sided Wilcoxon signed rank test $p=2.4 \times 10^{-55}$, test statistic $=472$, Fig. $2 f$, Supplementary Data 1).

150 Additionally, we examined the central coverage for the 338 TFs in a cohort of 215 healthy donors ${ }^{38}$

151 before and after GC correction. Because healthy donor samples have no tumor content, we 152 evaluated the mean absolute deviation (MAD) for each TF to compare inter-sample variability.

153 We found that the MAD decreased after GC correction for 324 (95.8\%) TFs (two-sided Wilcoxon 154 signed rank test $p=1.4 \times 10^{-53}$, test-statistic $=940$, Fig. $2 \mathrm{~g}$, Supplementary Data 2), indicating 155 lower inter-sample variability for nearly all TFs. Altogether, these results suggest that the novel 156 GC correction in the Griffin framework reduces the variability in chromatin accessibility signals 157 due to GC biases between samples and allows for improved detection of tissue specific accessibility in ULP-WGS data.

\section{Griffin analysis at TFBS enables accurate cancer detection and tissue-of-origin prediction}

161 To determine if Griffin can perform cancer detection, we analyzed a published WGS (1-2X)

162 dataset of cfDNA samples from healthy donors $(n=215)$ and cancer patients $(n=208) .{ }^{38}$ We

163 generated nucleosome profiles around TFBSs for the 338 TFs using nucleosome sized (100-

164 200bp) fragments and extracted three features from each profile (central coverage, mean

165 coverage, and amplitude) for a total of 1014 features. Using logistic regression, we achieved a 166 high performance for predicting the presence of cancer with an area under the receiver operating 167 curve (AUC) of 0.96 (Fig. 3a, Supplementary Data 3). We achieved the highest prediction 168 performance for lung and ovarian cancers $(A \cup C=1.00)$ and the lowest for pancreatic cancer 
$169(\mathrm{AUC}=0.90)$. We also observed high performance for stage IV cancers (AUC=0.99) but

170 maintained great performance for stage I cancers (AUC=0.94, Fig. Supplementary Fig. 3). The

171 performance was likely reflective of the higher tumor fractions observed in late-stage cancer

172 relative to early-stage cancer. We observed higher performance for samples with tumor fraction

$173 \geq 0.05$ (AUC 1.0) than samples with undetectable tumor (0 tumor fraction, AUC=0.94,

174 Supplementary Fig. 3). We also observed similar performance with Griffin analysis around DNase

175 I Hypersensitivity Sites (DHS) (AUC=0.91, Supplementary Fig. 3).

176

177 To test the ability to detect cancer at ULP-WGS coverage (0.1x), we applied Griffin to the same

178 cfDNA data downsampled to $0.1 x$ coverage and achieved a performance with AUC of 0.88 (Fig.

$1793 b)$. Next, because fragments $<150 \mathrm{bp}$ are enriched for tumor derived DNA ${ }^{38}$, we tested whether

180 using only shorter fragments might improve our ability to detect cancer in this framework, we

181 applied Griffin to analyze only 35-150bp fragments at the same TFBSs and observed a decreased

182 performance $(A \cup C=0.93$, Supplementary Fig. 3). Finally, we compared our results with the

183 method by Ulz et al. ${ }^{42}$, which analyzed cfDNA fragments of all lengths at TFBSs. Across all cancer

184 types, Griffin using nucleosome-sized or short fragments and ULP-WGS coverage had higher

185 detection performance (Fig. 3c, Supplementary Fig. 3). This demonstrates that Griffin can detect

186 cancer accurately using various sites from chromatin-based assays and cost-effective ULP-WGS

187 of cfDNA.

188

189 Next, we tested the ability of Griffin to predict the cancer tissue of origin from cfDNA. Using Griffin

190 nucleosome profile features around the TFBSs for the 338 TFs, we applied a multinomial logistic

191 regression to predict the cancer type of each sample. The top prediction was correct for $60 \%$ of

192 samples. When the top two predictions were considered, $79 \%$ of the samples were correctly

193 classified (Fig. 3d). Overall, we show that Griffin can be used for highly accurate cancer detection 
194 from cfDNA even when using ULP-WGS coverage and that Griffin can be used for tissue of origin

195 prediction.

\section{Griffin enables accurate prediction of breast cancer subtypes from ultra-low pass WGS}

198 Breast cancer tumor classification relies on accurate clinical determination of hormone receptor 199 status primarily by immunohistochemistry (IHC) to quantify the expression of ER, but no ctDNA 200 approach exists for this application. We set out to determine whether Griffin can be used to predict 201 ER subtype status from ULP-WGS (0.1x) of cfDNA from MBC patients. We analyzed 254 202 samples ${ }^{10,11}$ with tumor fraction greater than 0.05 from 139 patients. First, we inspected the Griffin 203 profiles at TFBSs for key factors, including ESR1, FOXA1, and GATA3, which are known to be associated with ER positive tumors. ${ }^{46}$ We observed that these TFBSs were more accessible in cfDNA samples from patients with ER+ metastases compared to ER-; central coverage was negatively correlated with tumor fraction for ER+ samples only (Pearson's $r<-0.35, p<4.2 \times 10^{-4}$,

207 Supplementary Fig. 4). To predict ER status, we initially built a logistic regression classifier using features from the Griffin profiles for all 338 TFs and achieved an accuracy of 0.68 (AUC of 0.74, subtyping and observed an accuracy of 0.55 (AUC=0.58, Supplementary Fig. 5), likely because

211 it was not designed for ULP-WGS data.

213 Next, we used a more tailored site selection approach by analyzing regions of differential

214 chromatin accessibility. Using ATAC-seq data generated from $44 \mathrm{ER}+$ and $15 \mathrm{ER}$ - primary breast

215 tumors by The Cancer Genome Atlas (TCGA) ${ }^{47}$, we identified open chromatin sites that were 216 specific to each ER subtype (Methods, Fig. 4a, Supplementary Data 4). ER+ specific sites $217(n=27,359)$ were enriched for the TFBSs of ESR1, PGR, FOXA1 and GATA3, and ER- specific 218 sites $(n=24,861)$ were enriched for the TFBSs of STAT3 and NFKB1 (Supplementary Data 5). We 219 observed differences in coverage profiles between ER subtype-specific sites that were shared 
and not shared with accessible chromatin in hematopoietic cells ${ }^{48}$ and analyzed them separately

221 (Fig. 4b, Supplementary Fig. 6).

222

223 We applied Griffin to profile nucleosome accessibility at these four sets of ER subtype-specific

224 accessible chromatin sites, extracting a total of 12 features (Fig. 4b, Supplementary Fig. 6). We

225 built a logistic regression classifier to predict ER subtype from these chromatin accessibility

226 features and achieved an overall accuracy of 0.81 ( $A \cup C=0.89, n=139$ ) (Methods, Fig. 4c). The

227 performance was higher for samples with high tumor fraction (accuracy 0.88, AUC=0.93, n=101,

228 tumor fraction $\geq 0.1$ ) compared to those with lower tumor fraction (accuracy 0.64 , AUC=0.68,

$229 \mathrm{n}=38$, tumor fraction 0.05 to 0.1 ) (Fig. 4c). Repeating the analysis using only short fragments (35-

$230150 \mathrm{bp}$ ) did not improve the performance (accuracy 0.66, AUC=0.71), likely due to further reduced

231 fragment coverage (Supplementary Fig. 5). These results illustrate the utility of using chromatin

232 accessibility for cancer subtyping from ULP-WGS data and showcase the first application of ER

233 status prediction in breast cancer from cfDNA.

\section{Analysis of ER status from cfDNA reveals tumor subtype heterogeneity}

236 To further investigate the ER predictions, we inspected the classification results for 48 of the

237 patients with an ER- metastasis, known primary ER status, and a tumor fraction of $\geq 0.1$. In 41

238 patients with where the primary and metastasis were both ER- by IHC, we predicted $39(95.1 \%)$

239 patients to have ER-subtype. Intriguingly, in the seven patients who had clinical primary ER+ and

240 metastatic ER- status (i.e., ER loss), three (42.9\%) were predicted to be ER+, and this higher

241 prevalence of ER+ prediction for this patient group was statistically significant (two-sided Fisher's

242 exact test $p=0.018$, Fig. 4d). We also observed that the predicted probability of ER+ was higher

243 in the patients with ER loss than the patients with ER-primary and metastasis, and this was

244 statistically significant even after accounting for tumor fraction (analysis of covariance, $p=0.014$ ). 
245 These results suggest that there may be residual ER+ tumor features in the ER loss patients or

246 that Griffin analysis may be capturing a heterogeneous mixture of ER subtypes from ctDNA.

248 To further assess whether this observation may be due to tumor heterogeneity, we examined

249 ULP-WGS samples from six TNBC patients receiving treatment with Cabozantinib who had

250 plasma collected at different timepoints and had clonal dynamics analysis performed previously

251 using subclonal somatic mutations from ctDNA. ${ }^{11,49}$ Overall for all six patients, the ER+ probability

252 followed closely to the trends of multiple clones over time (Fig. 4f, Supplementary Fig. 7). In

253 patient MBC_1306, ER+ probability tracked closely with the clonality of clonal cluster 4, as

254 estimated by the cellular prevalence ${ }^{50}$, particularly at 21.7 months post-metastasis where both

255 increased (Fig. 4f). Two of these six patients (MBC_1413 and MBC_1405) had known ER loss

256 for at least one metastasis. Interestingly in both cases, the ER+ probability fluctuated over time,

257 but tracked with one or more of the genomic clones (Fig. 4f). In patient MBC_1413, who had an

258 ER+ primary and ER- metastasis, we noted the ER+ probability tracked closely with the cellular

259 prevalence of clonal cluster 3, including the coincident $0.4 \mathrm{ER}+$ probability increase with a $30 \%$

260 (cluster 3) expansion at 10 months post-metastasis (Fig. 4g). Patient MBC_1405 had an ER+

261 primary and both ER- and ER+ metastatic biopsies but was considered ER+ status despite having

262 only $25 \%$ expression by IHC. While all five timepoints from this patient were predicted to be ER-,

263 the ER+ probability tracked with both clonal clusters 3 and 4 . Furthermore, the proximity of the

264 predicted ER+ probabilities near the decision boundary suggests we may be capturing the

265 heterogeneity of the two metastatic biopsies. These results support the presence of ER subtype

266 heterogeneity as compared with orthogonal ctDNA clonality analysis and suggest that tumor

267 subtype dynamics can be monitored during therapy. 


\section{Discussion}

272 In this study, we described the development of Griffin, a new framework and analysis tool for

273 studying transcriptional regulation and tumor phenotypes. Griffin uses a novel cfDNA fragment

274 length-specific normalization of GC-content biases that obscure chromatin accessibility

275 information. We demonstrated that Griffin can be used to detect cancer from low pass WGS with

276 high accuracy. We also developed an approach to perform ER subtyping in breast cancer from

277 ULP-WGS, which to our knowledge is the first time that ER phenotype prediction has been shown

278 from ctDNA.

279

280 Griffin is versatile and can be used for various applications in cancer. We highlighted cancer 281 detection, tissue-of-origin, and tumor subtype use-cases. However, Griffin can also be used for 282 any biological comparison where transcriptional regulation and chromatin accessibility differences

283 can be delineated. The applications described here use TFBSs from chromatin 284 immunoprecipitation sequencing (ChIP-seq) and accessible chromatin sites from ATAC-seq. 285 However, Griffin differs from existing methods due to its ability to analyze custom sites of interest 286 that are specific to any biological context. These sites may be obtained from external sources and 287 different assays, such as ChIP-seq, DNase I hypersensitivity, ATAC-seq or cleavage under 288 targets and release using nuclease (CUT\&RUN). As additional epigenetic data are collected by 289 the cancer research community, including from single-cell experiments ${ }^{51,52}$, Griffin will be integral 290 for advancing tumor phenotype studies from liquid biopsies.

291

292 Griffin is optimized for the analysis of ULP-WGS (0.1x) of cfDNA, while other nucleosome profiling 293 methods have focused on deeper coverage sequencing. Griffin takes advantage of analyzing the 294 breadth of sites as opposed to individual loci, which was inspired by a similar strategy used by 295 Ulz et $\mathrm{al}^{42}$. We show that Griffin has better performance for both detecting cancer and predicting 296 ER status from ULP-WGS data when compared to the Ulz method, because of its novel bias 
correction and versatility to analyze any set of genomic regions. However, Griffin is not limited to low coverage data. Increased cfDNA sequencing coverage can allow for analysis of specific gene promoters and cis-regulatory elements and may be able to inform gene expression. ${ }^{31}$ While recent studies show the promise of cfDNA methylation and cfRNA analysis for tumor phenotype analysis and cancer detection, ${ }^{53-59}$ these analytes may be challenging to isolate from clinical specimens or require specialized assays. Griffin provides a cost-effective and scalable method requiring only standard low coverage WGS of cfDNA, which can be more rapidly incorporated into existing platforms to predict clinical cancer phenotypes.

A limitation of the binary ER classification (ER+ or ER-) is the decreased accuracy for samples with lower tumor fraction (0.05 to 0.1$)$; however, patients with cfDNA tumor fraction $\geq 10 \%$ have poorer prognosis ${ }^{60}$ and would benefit more from tumor monitoring. It may be possible to improve performance of ER subtyping for lower tumor fraction samples with additional sequencing depth or joint analysis of multiple cfDNA timepoints from the same patient.

312 The application of Griffin to predict ER status from cfDNA of MBC patients led to interesting

313 insights into tumor heterogeneity and potential explanations for misclassified predictions.

314 Intriguingly, we noticed that for the patients with ER- tumors by IHC, ER+ predictions were 315 significantly enriched when the primary tumor was ER+. Moreover, we observed that the predicted 316 ER probability closely matched the clonal dynamics from somatic mutation in six patients. Two of 317 these patients had a change in predicted ER status, potentially suggesting the presence of 318 metastases of both subtypes. Importantly, while this subtype heterogeneity and switching would 319 typically not be captured from a single metastatic biopsy, our results demonstrate the possibility 320 of using ER probability to monitoring subtype heterogeneity over time during therapy using ctDNA. 
322 We focus our breast cancer subtyping on ER prediction because its status has important utility in

323 predicting likely benefit to endocrine therapy. ${ }^{61}$ While PR expression is also determined in the

324 clinic and ER-/PR+ tumors are considered hormone receptor positive, these are rare, not

325 reproducible or less useful for prognosis. ${ }^{62}$ In our cohort, only 2 of $139(1.4 \%)$ patients were ER-

326 /PR+. HER2 overexpression is important relevant for prognosis and determining treatment such

327 as trastuzumab. ${ }^{63}$ However, we were unable to identify sufficient number of open chromatin sites

328 that were specific for distinguishing HER2 status. Since ERBB2 (encodes the HER2 protein) is

329 amplified in $\sim 20 \%$ breast cancers, one can instead assess ERBB2 copy number amplification

330 from ctDNA genomic analysis. ${ }^{64}$ Alternatively, a model to predict PAM50 status could be useful

331 as this may be a better indicator of prognosis than ER/PR/HER2 IHC alone. ${ }^{65}$

333 The Griffin framework is a unique advance on our previous method to analyze genomic alterations

334 and estimate tumor fraction from ULP-WGS of cfDNA. ${ }^{10}$ Together, these methods form a suite of

335 tools to establish a new paradigm to study both tumor genotype and phenotype from ULP-WGS

336 of cfDNA. Griffin has the potential to reveal clinically relevant tumor phenotypes, which will support

337 the study of therapeutic resistance, inform treatment decisions, and accelerate applications in

338 cancer precision medicine.

$340 \quad$ Methods

341 Griffin: Site filtering

342 Prior to performing nucleosome profiling, we filtered all site lists by mappability to remove regions

343 that had low or uneven coverage due to inability to map reads. We used mappability data from

344 the hg38 Umap multi-read mappability track for 50bp reads downloaded from the UCSC genome

345 browser $^{66}$ (downloaded from

346 https://hgdownload.soe.ucsc.edu/gbdb/hg38/hoffmanMappability/k50.Umap.MultiTrackMappabili

347 ty.bw). To perform this filtration, we developed the 'griffin_filter_sites' pipeline. This pipeline takes 

calculated the mean mappability value using pyBigWig (https://github.com/deeptools/pyBigWig). We then excluded sites with a mean mappability below the threshold of 0.95 and retained highly mappable sites for further analysis.

\section{Griffin: GC bias calculation}

355 GC content influences the efficiency of amplification and sequencing leading to different expected 356 coverages (coverage bias) for fragments with different GC contents and fragment lengths. This is called GC bias and is unique to each sample. We calculated the GC bias of each bam file using a custom method similar to that demonstrated in Benjamini and Speed $2012^{44}$ and implemented in deepTools ${ }^{67}$. However, unlike this existing approach, which assumes that all fragments have the same length, our approach calculates a separate GC bias curve for every fragment length within a specified range. This is helpful for cfDNA where different samples may have different fragment size distributions. Prior to performing GC bias calculation, we identified all mappable, non-repetitive regions of the genome. We used pybedtools to find the mappable regions (defined as mappability score = 1) from the hg38 mappability track (described in the section on site filtering) and exclude the repetitive regions from the UCSC hg38 repeat masker track ${ }^{68}$ (downloaded from the UCSC table browser: http://genome.ucsc.edu/cgi-bin/hgTables). We then examined all

367 mappable, non-repetitive regions of the genome and, for each fragment length, counted the 368 number of times each GC content is observed in possible fragments overlapping those regions. 369 These counts for each fragment length are the 'genome GC frequencies'. We then developed the 370 'griffin GC bias' pipeline to compute the GC bias in a given bam file. The pipeline takes a bam 371 file, bedGraph file of valid (mappable, non-repetitive) regions, and genome GC frequencies for

372 those regions. For each given sample, we fetched all reads aligning to mappable, non-repetitive 373 regions on autosomes using pysam (https://github.com/pysam-developers/pysam) ${ }^{69}$. We counted 
374 the number of observed reads for each length and GC content, excluding reads with low mapping

375 quality $(<20)$, duplicates, unpaired reads, and reads that failed quality control. These read counts

376 are the 'GC counts' for that sample. We then divided the GC counts by the GC frequencies to

377 obtain the GC bias for that bam file and normalized the mean GC bias for each fragment length

378 to 1, resulting in a GC bias value for every combination of fragment size and GC content (except

379 those that are not observed in the genome). We then smoothed the GC bias curves. For each

380 fragment size we took all GC bias values for fragments of a similar length (+/- $10 \mathrm{bp})$. We sorted

381 these values by the GC content of the fragment to create a vector of GC bias values for similar

382 sized fragments. We then smoothed this vector by taking the median of $\mathrm{k}$ nearest neighbors

383 (where $k=5 \%$ of the vector length or 50 , whichever is greater) and repeated for each possible

384 fragment length. We then normalized to a mean GC bias of 1 for each possible fragment length

385 to generate a smoothed GC bias value for every possible fragment length and GC content observed in the genome.

\section{Griffin: Nucleosome profiling}

389 We designed the griffin nucleosome profiling pipeline to perform nucleosome profiling around 390 sites of interest. This pipeline takes a bam file and site list, and assorted other parameters 391 described below. For a given bam file and site list, we fetched all reads in a window ( -5000 to $392+5000 \mathrm{bp})$ around each site using pysam (excluding those that failed quality control measures). 393 We then filtered reads by fragment length and selected those in a range of fragment lengths 394 (typically 100-200 bp unless otherwise specified). For each read, we determined the GC bias for 395 each fragment and assigned a weight of $\frac{1}{G C \text { bias }}$ to that fragment and identified the location of the 396 fragment midpoint. We split the site into $15 \mathrm{bp}$ bins and summed the weighted fragment midpoints 397 in each bin to get a GC corrected midpoint coverage profile (see Fig. $1 \mathrm{~b}$ for a schematic). We 398 repeated this for every site on the site list and took the mean of all sites to generate the coverage 
profile for that site list. To make samples with different depths comparable, we normalized the coverage profile to a mean coverage of 1 . We then smoothed the coverage profiles using a 401 Savitzky-Golay filter with window length 165bp and polynomial order of 3.

\section{Griffin: Nucleosome profile feature quantification}

404 To quantify coverage profiles, we extracted 3 features from each coverage profile. First, we 405 calculated the 'mean coverage' value +/- $1000 \mathrm{bp}$ from the site. Second, we calculated the 406 coverage value at the site (+/-30bp). And third, we calculated the amplitude of the nucleosome 407 peaks surrounding the site by using a Fast Fourier Transform (as implemented in Numpy ${ }^{70}$ ) on 408 the window $+/-960 \mathrm{bp}$ from the site and taking the amplitude of the $10^{\text {th }}$ frequency term. This window and frequency were chosen due to the observed nucleosome peak spacing at an active site (190bp) which results in approximately 10 peaks in the window +/-960bp.

\section{Early-stage cancer and healthy donor cfDNA samples}

413 Whole genome sequencing (WGS) cfDNA from patients with various types of early stage cancer 414 and healthy donors were obtained from an existing dataset published in Cristiano et al ${ }^{38}$. Bam 415 files were downloaded from EGA (dataset ID: EGAD00001005339). This data consisted of 1-2x 416 low pass whole genome sequencing from $100 \mathrm{bp}$ paired end Illumina sequencing reads. For our 417 analyses, we used a subset of samples with 1-2X WGS of cfDNA from 208 cancer patients with 418 no previous treatment and 215 healthy donors. These are the samples used for the cancer 419 detection analysis in Cristiano et al. cfDNA tumor fraction was estimated using ichorCNA ${ }^{10}$. An 420 hg38 panel of normal (PoN) with a $1 \mathrm{mb}$ bin size was created using all 260 healthy donors in the 421 dataset. ichorCNA was then run on all cancer and healthy samples to estimate tumor fraction. 422 ichorCNA_fracReadsInChrYForMale was set to 0.001. Defaults were used for all other settings. 
425 WGS of cfDNA from patients with metastatic breast cancer (MBC) and healthy donors were

426 obtained from an existing dataset published by Adalsteinsson and colleagues ${ }^{10}$. Bam files were

427 downloaded from dbGaP (accession code: phs001417.v1.p1). This data consisted of $\sim 0.1 \mathrm{x}$ ultra-

428 low pass whole genome sequencing (ULP-WGS) from 100bp paired end Illumina sequencing

429 reads. For our analyses, we used a subset of 254 samples with $>0.1 \mathrm{X}$ coverage WGS, $>0.05 \mathrm{X}$

430 tumor fraction and known estrogen receptor (ER) status. Of these 254 samples 132 were ER

431 positive (from 74 unique patients) and 122 were ER negative (from 65 unique patients). Coverage

432 and tumor fraction metrics were obtained from the supplemental data in the publication ${ }^{10}$. Primary

433 and metastatic ER and PR status determined by immunohistochemistry. Additionally, we used

434 deep (9-25X) WGS from two MBC patients (MBC_315 and MBC_288) from the same source and

435 deep (17-20X) WGS from two healthy donors (HD45 and HD46) from the same source for 436 designing and demonstrating the pipeline.

438 For training and assessing the ER status classifier we labeled each sample as ER+ or ER- using 439 information about the ER status from medical records. If metastatic ER status was known, the 440 sample was labeled according to this status. If metastatic ER status was not known, the sample

441 was labeled according to the primary tumor ER status (20 samples from 11 patients). ER low 442 samples (9 samples from 5 patients) were labeled ER positive for the purpose of the binary 443 classifier. For three patients (MBC_1405, MBC_1406, MBC_1408), we had information about 444 multiple metastatic biopsies with different ER statuses. In these cases, we used the last biopsy 445 taken for the purpose of the binary ER status classifier.

\section{Human Subjects}

448 WGS of cfDNA samples from patients with MBC were obtained from an existing study as 449 described above ${ }^{10}$. Additional information, including primary ER status, metastatic ER status, and 450 survival time, was abstracted from the medical records. Use of this data was approved by an 
451 institutional review board (Dana-Farber Cancer Institute IRB protocol identifiers 05-246, 09-204,

452 12-431 [NCT01738438; Closure effective date 6/30/2014]).

\section{Sequence data processing}

455 All sequencing data used in this study was realigned to the hg38 version of the human genome 456 (downloaded from http://hgdownload.soe.ucsc.edu/goldenPath/hg38/bigZips/hg38.fa.gz). Bam

457 files were unmapped from their previous alignment using Picard SamToFastq. ${ }^{71}$ They were then 458 realigned to the human reference genome according to GATK best practices ${ }^{72}$ using the following 459 procedure. Fastq files were realigned using BWA-MEM. ${ }^{73}$ Files were then sorted with samtools ${ }^{74}$, 460 duplicates were marked with Picard, and base recalibration was performed with GATK, using 461 known polymorphisms downloaded from the following locations:

462 https://console.cloud.google.com/storage/browser/genomics-public-

8.vcf.gz.

\section{Transcription factor binding site (TFBS) selection}

468 Transcription factor binding sites (TFBSs) were downloaded from the GTRD database ${ }^{45}$. This 469 database contains a compilation of ChIP seq data from various sources. For our analyses, we 470 used the meta clusters data (version 19.10, downloaded from

471 https://gtrd.biouml.org/downloads/19.10/chip-seq/Homo\%20sapiens_meta_clusters.interval.gz).

472 This contains meta peaks observed in one or more ChIP seq experiments. The GTRD database 473 contains some ChIP seq experiments for targets that are not transcription factors (TFs). These 474 were excluded by comparing against a list of TFs with known binding sites in the CIS-BP 475 database ${ }^{75}$ (v2.00 downloaded from http://cisbp.ccbr.utoronto.ca/bulk.php). TFBS were then 476 filtered by mappability as described above (Griffin: Site Filtering). The site position was identified 
477 as the mean of 'Start' and 'End'. TFs with less than 10,000 highly mappable sites on autosomes

478 were excluded. For each remaining TF, the top 10,000 highly mappable sites were selected by

479 choosing those with the highest 'peak.count' (number of times that peak has been observed

480 across all experiments).

481

482 DNase I hypersensitivity site selection

483 DNase I hypersensitivity sites for a variety of tissue types were downloaded from 484 https://zenodo.org/record/3838751/files/DHS Index and Vocabulary hg38 WM20190703.txt.g

$485 \quad \underline{z}^{76}$. These sites were split by tissue type for a total of 16 site lists. They were filtered by mappability 486 as described above (Griffin: Site Filtering) using the 'summit' column as the site position. The 487 highly mappable sites were sorted by the number of samples where that site had been observed 488 ('numsamples') and the top 10,000 most frequently observed sites were selected for each tissue 489 type.

\section{$491 \quad$ ATAC-seq site selection for ER subtyping}

492 Assay for transposase-accessible chromatin using sequencing (ATAC-seq) site accessibility for 493 primary breast cancer samples from The Cancer Genome Atlas (TCGA) were downloaded from 494 the TCGA ATAC-seq $\quad$ hub 495 (https://atacseq.xenahubs.net/download/brca/brca peak Log2Counts dedup $)^{47}$. The locations 496 of these sites and patient metadata were obtained from the supplemental tables in the paper ${ }^{47}$. 497 These ATAC-seq sites were filtered for mappability as described above (Griffin: Site Filtering), 498 using the mean of the Start and End columns as the peak position. High mappability sites on 499 autosomes were kept for further analysis for a total of 142,490 sites. Differentially accessible sites 500 between ER+ $(n=44)$ and ER- $(n=15)$ tumors were identified by using a Mann-Whitney $U$ test. P 501 values were corrected for multiple testing using the Benjamini/Hochberg procedure using 502 statsmodels ${ }^{77}$ and sites with a q-value $<0.05$ were selected. Additionally, selected sites were 
503 further filtered based on the log2 fold change between ER+ and ER- tumors. Sites with a log2 fold

504 change $>0.5$ were classified as ER+ specific, while sites with a log2 fold change <-0.5 were

505 classified as ER- specific. These site lists were further split into sites shared with hematopoietic

506 cells and those not shared with hematopoietic cells. Hematopoietic sites were obtained from a

507 database of single cell ATAC-seq data ${ }^{48}$ (GEO accession number: GSE129785, peak file

508 available

here:

509 https://ftp.ncbi.nlm.nih.gov/geo/series/GSE129nnn/GSE129785/suppl/GSE129785\%5FscATAC

$510 \quad \% 2 \mathrm{DH}$ Hematopoiesis\%2DAll\%2Epeaks\%2Etxt\%2Egz). Hematopoietic peaks were lifted over to

511 hg38 using the UCSC liftover command line tool and sites that changed size during liftover $(0.2 \%$

512 of peaks) were discarded. BRCA ATAC-seq sites that overlapped with Hematopoietic sites

513 (Overlapping peaks were defined as site centers being within 500bp of one another) this was

514 performed using pybedtools intersect ${ }^{78,79}$. This resulted in a total of 4 differential site lists: ER

515 positive sites that were not shared with hematopoietic cells $(15,142$ sites), ER positive sites that

516 were shared with hematopoietic cells (12,217 sites), ER negative sites that were not shared with

517 hematopoietic cells (12,151 sites), and ER negative sites that were shared with hematopoietic

518 cells $(12,710$ sites $)$.

519 We then overlapped these differential ATAC-seq site lists with the top 10,000 sites for each of

520338 transcription factors (TFs) using pybedtools intersect. An overlapping pair of sites was defined

521 as having $<500 \mathrm{bp}$ between site centers. Each differential ATAC-seq site list was compared

522 against each list of TFBSs and the total number of ATAC sites overlapping one or more TFBS on

523 the given list was recorded.

524

\section{Assessment of Griffin before and after GC correction}

526 Tumor fraction correlations at TFBS

527 For $191 \mathrm{MBC}$ ULP samples with $>0.1$ tumor fraction, nucleosome profiling with and without GC 528 correction was performed on the top 10,000 sites for each of 338 transcription factors (TFs). For 
each TF, the relationship between central coverage and tumor fraction was modeled using

530 scipy.stats.linregress ${ }^{80}$ producing a Pearson correlation ( $r$ ) and line of best fit. Root mean squared

531 error (RMSE) was calculated from the line of best fit. This was performed both before and after

532 GC correction as illustrated for Lyl-1 in Fig. 2e. For all 338 TFs, the RMSE values before and after

533 GC correction were compared using a Wilcoxon signed-rank test (two-sided).

Mean absolute deviation (MAD) at TFBS

536 For 215 healthy donors, nucleosome profiling with and without GC correction was performed on

537 the top 10,000 sites for each of 338 TFs. For each TF, the MAD of the central coverage values 538 was calculated both before and after GC correction. For all 338 TFs, the MAD values before and after GC correction were compared using a Wilcoxon signed-rank test (two-sided).

\section{Machine learning, bootstrapping, and performance evaluation procedure}

542 To detect cancer, predict tissue type, or predict ER subtype, we used logistic regression with

543 Ridge regularization (i.e. L2 norm) as implemented in scikit-learn ${ }^{81}$. All feature values were scaled

544 to a mean of 0 and a standard deviation of 1 prior to performing bootstrapping and fitting the

545 models. We used the following bootstrapping procedure to train and assess the performance of

546 our models. First, we selected $\mathrm{n}$ samples with replacement from the full set of $\mathrm{n}$ samples and

547 used this as a training set. Samples that weren't selected were used as the test set. We then used

54810 -fold cross-validation on the training set to select the parameter ' $C$ ' (inverse of the regularization

549 strength) from the following options: $10^{-5}, 10^{-4}, 10^{-3}, 10^{-2}, 10^{-1}, 10^{0}, 10^{1}, 10^{2}$. To account for class

550 imbalances in the data we used set the 'class weight' parameter to 'balanced' to adjust the sample

551 weighs inversely proportional to the class frequencies. We trained a final model on all the training

552 data using the selected regularization strength. Finally, we tested this model on the test set and

553 recorded the performance (accuracy and AUC values) and probabilities from each sample. Then,

554 a new training set was selected, and the procedure was repeated for 2000 iterations (for cancer 
555 detection and tissue of origin analysis) or 1000 iterations (for breast cancer subtyping). After

556 completing the bootstrap iterations, we calculated the AUC and accuracy from each bootstrap

557 iteration and used these to generate the mean and 95\% confidence interval around each of these

558 values. To visualize the mean ROC curve, we used the median probability from all bootstraps

559 where that sample was included in the test set. For further downstream analyses, we used this

560 same median probability.

561

\section{Features used for cancer detection classification}

563 To detect cancer, we applied the logistic regression approach described above and built four

564 different models using four different sets of features extracted from the pan cancer patient 565 samples and healthy donor samples. First, we performed nucleosome profiling in these samples 566 (selecting fragments 100-200bp in length) on the 338 selected TFs from the GTRD database. We

567 extracted three features (as described above) from each coverage profile for a total of 1,014 568 features.

569 Second, we performed nucleosome profiling on these same samples and sites but selected only 570 'short' fragments (35-150bp) to be counted in the nucleosome profiles.

571 Third, we downsampled these samples to $\sim 0.1 \mathrm{x}$ coverage (procedure described below) and

572 performed nucleosome profiling for the same 338 TFs selecting fragments 100-200bp in length.

573 Fourth, we used the original (not downsampled) samples and performed nucleosome profiling at

574 the 16 tissue-specific DHS site lists described above. We extracted the same 3 features from 575 each site profile for a total of 48 features.

576

\section{Tissue of origin prediction}

578 For tissue of origin prediction, we used the nucleosome profiles from the 338 TFs in the 1-2X 579 coverage (not downsampled) cancer samples using 100-200bp fragments. We excluded 1 580 duodenal cancer sample as this was the only sample from that cancer type. This left us with 207 
581 cancer samples from 7 different cancer types: bile duct $(n=25)$, breast $(n=54)$, colorectal $(n=27)$,

582 gastric $(n=27)$, lung $(n=12)$, ovarian $(n=28)$, and pancreatic $(n=34)$. We built a multinomial logistic

583 regression model to predict the cancer tissue of origin for each sample using the same

584 bootstrapping strategy described above. We ran this for 2000 iterations. For each iteration, we

585 calculated the accuracy of the top prediction as well as the top two predictions.

587 Downsampling of pan-cancer and healthy donor cfDNA sequencing data

588 1-2x WGS of pan-cancer patient and healthy donor bam files aligned to hg38 were downsampled 589 using Picard DownSampleSam. The probability used by DownSampleSam was calculated based 590 on a target of $2,463,109$ read pairs which resulted in approximately $0.11 x$ coverage as calculated 591 by Picard CollectWgsMetrics. Downsampled bam files were realigned to hg19 for use in the Ulz 592 pipeline. The realignment procedure was the same as above but using the hg19 genome 593 (downloaded from https://hgdownload.soe.ucsc.edu/goldenPath/hg19/bigZips/hg19.fa.gz) and 594 hg19 known polymorphic sites for base recalibration (downloaded from ftp://gsapubftp595 anonymous@ftp.broadinstitute.org/bundle/hg37/Mills and 1000G gold standard.indels.hg37.v

597 ftp://ftp.ncbi.nih.gov/snp/organisms/human 9606 b151 GRCh37p13/VCF/GATK/All 20180423. $598 \quad$ vcf.gz).

\section{ER status classification in the MBC cohort}

601 To predict ER status, we applied the logistic regression approach described above to features 602 extracted from the MBC patient samples. Because some patients had multiple samples, we 603 modified the bootstrapping procedure to select 139 patients (rather than samples) with 604 replacement from a full set of 139 patients. For each selected patient, all samples from that patient 605 were added to the training set (If a patient was selected multiple times, all their samples were 606 included multiple times). This ensured that separate samples from the same patient (biological 
replicates) could not appear in both the training and test set. Samples from patients that weren't selected were used as the test set.

610 Using these training and tests sets, we built three different models based on three different sets

611 of features. First, we applied nucleosome profiling using 100-200bp fragments to the 338 TFs

612 from GTRD and extracted 3 features per profile for a total of 1014 features. Second, we applied

613 nucleosome profiling using 100-200bp fragments to the 4 ER differential ATAC seq lists and

614 extracted 3 features per profile for a total of 12 features. Lastly, we applied nucleosome profiling

615 using 35-150bp fragments to the 4 ER differential ATAC seq lists and extracted 3 features per list

616 for a total of 12 features.

618 For evaluating the models, we only included the first timepoint for each patient in the test set when calculating the accuracy and AUC for each bootstrap iteration. This prevented a small number of patients with many samples from having a large impact on the scores.

ER probability comparison between patients with and without ER loss using analysis of

624 To determine whether the probability of ER+ for the patients with ER loss (primary ER+, metastatic

625 ER-) were significantly different from the probability of ER+ for the patients with ER- primary and

626 metastasis disease, we performed an analysis of covariance (ANCOVA) as implemented in

627 Pingouin ${ }^{82}$. Probability of ER+ was the dependent variable, primary tumor status was the 628 independent variable ('between'), and tumor fraction was a covariate. While we found that tumor 629 fraction was significantly related to the ER probability $(p=0.03, F=5.02$, degrees of freedom $=1)$, 630 we also found a significant difference $(p=0.014, F=6.48$, degrees of freedom $=1)$ between the 631 ER loss and ER- unchanged patients. 


\section{Transcription factor profiling using pipeline from Ulz et al.}

634 We downloaded the Transcription Factor Profiling pipeline published by Ulz and colleagues from

635 Github (https://github.com/PeterUlz/TranscriptionFactorProfiling) ${ }^{42}$ and ran it using the following

636 procedure as described in the paper. hg19 aligned bam files were used because the pipeline was

637 written to for this version of the genome. Scripts were modified so that they worked in python3.

638 We trimmed the reads in each bam to 60bp using 'trim from bam single end' with modifications to 639 skip unaligned reads. We ran ichorCNA on the original (untrimmed) bam using the default 640 ichorCNA settings for hg19 except the bin size, which was modified to 50,000bp and no panel of 641 normals. We then ran the transcription factor profiling analysis on the trimmed bam using the 642 script run_tf_analyses_from_bam.py with options '-calccov' and '-a tf_gtrd_1000sites' and the 643 ichorCNA corrected depth file as the '-norm-file'. This ran transcription factor profiling on 1,000 644 sites for each of 504 TFs. Finally, we ran the scoring pipeline. We used the high frequency 645 amplitude ('HighFreqRange') for each of the 504 TFs in the accessibility output file 646 (Accessibility1KSitesAdjusted.txt) as the features for a logistic regression model using the same 647 bootstrapping scheme described above.

\section{Clonality analysis}

650 For 6 patients with high tumor fractions, multiple samples, and triple negative breast cancer, data 651 on clonal dynamics in the ctDNA was available from a previous study ${ }^{49}$ (results downloaded from:

652 https://gitlab.com/Zt Weber/narrow-interval-clonal-structure-mbc/-/tree/master/PyClone-

653 Multisample-Final/pyclone output tables). In the study, somatic alterations were identified from 654 both WES and targeted panel sequencing using GATK-Mutect2. Using these alterations, clonal 655 dynamics were modeled using the $\mathrm{PyClone}^{50}$ package. The cellular prevalence estimate 656 represents the proportion of the sample that contains somatic mutation. PyClone reports clusters 657 of somatic mutations; cellular prevalence of these clusters is shown in the results. 


\section{Data availability}

660 Sequencing data used in this study was obtained from dbGaP (accession phs001417.v1.p1) and 661 EGA (dataset ID EGAD00001005339).

\section{Code availability}

664 Griffin software and the subtype classifier tool can be obtained from 665 https://github.com/adoebley/Griffin. Code for analysis and machine learning models can be 666 accessed at https://github.com/adoebley/Griffin analyses.

\section{References}

669 1. Heitzer, E., Auinger, L. \& Speicher, M. R. Cell-Free DNA and Apoptosis: How Dead Cells

670 Inform About the Living. Trends in Molecular Medicine 26, 519-528 (2020).

671 2. Diehl, F. et al. Circulating mutant DNA to assess tumor dynamics. Nature medicine 14, 985-

67290 (2008).

673 3. Maheswaran, S. et al. Detection of mutations in EGFR in circulating lung-cancer cells. The

$674 \quad$ New England journal of medicine 359, 366-77 (2008).

675 4. Wan, J. C. M. et al. Liquid biopsies come of age: towards implementation of circulating tumour DNA. Nature Reviews Cancer 17, 223-238 (2017).

677 5. Cohen, J. D. et al. Detection and localization of surgically resectable cancers with a multi678 analyte blood test. Science (New York, N.Y.) 359, 926-930 (2018).

679 6. McDonald, B. R. et al. Personalized circulating tumor DNA analysis to detect residual disease 680 after neoadjuvant therapy in breast cancer. Science Translational Medicine 11, eaax7392 $681 \quad$ (2019). 
7. Parsons, H. A. et al. Sensitive detection of minimal residual disease in patients treated for early-stage breast cancer. Clinical Cancer Research clincanres.3005.2019 (2020) doi:10.1158/1078-0432.ccr-19-3005.

8. Murtaza, M. et al. Non-invasive analysis of acquired resistance to cancer therapy by sequencing of plasma DNA. Nature 497, 108-112 (2014).

9. Zviran, A. et al. Genome-wide cell-free DNA mutational integration enables ultra-sensitive cancer monitoring. Nat Med 26, 1114-1124 (2020).

10. Adalsteinsson, V. A. et al. Scalable whole-exome sequencing of cell-free DNA reveals high concordance with metastatic tumors. Nature Communications 8, (2017).

691 11. Stover, D. G. et al. Association of Cell-Free DNA Tumor Fraction and Somatic Copy Number Alterations With Survival in Metastatic Triple-Negative Breast Cancer. Journal of Clinical

12. Choudhury, A. D. et al. Tumor fraction in cell-free DNA as a biomarker in prostate cancer.

$$
\text { JCl Insight 3, (2018). }
$$

13. Sumanasuriya, S. et al. Elucidating Prostate Cancer Behaviour During Treatment via Lowpass Whole-genome Sequencing of Circulating Tumour DNA. European Urology 80, 243-253 (2021). Biopsy in Prostate Cancer. JNCI: Journal of the National Cancer Institute 110, 78-86 (2018). Revealed by Linked-Read Genome Sequencing. Cell 174, 433-447.e19 (2018). 
16. Beltran, H. et al. Divergent clonal evolution of castration-resistant neuroendocrine prostate cancer. Nature Medicine 22, 298-305 (2016).

17. Bluemn, E. G. et al. Androgen Receptor Pathway-Independent Prostate Cancer Is Sustained through FGF Signaling. Cancer cell 32, 474-489.e6 (2017).

18. Aggarwal, R. et al. Clinical and Genomic Characterization of Treatment-Emergent Small-Cell Neuroendocrine Prostate Cancer: A Multi-institutional Prospective Study. JCO 36, 24922503 (2018).

19. Quintanal-Villalonga, A. et al. Multi-omic analysis of lung tumors defines pathways activated in neuroendocrine transformation. Cancer Discov (2021) doi:10.1158/2159-

20. Niederst, M. J. et al. RB loss in resistant EGFR mutant lung adenocarcinomas that transform 714 to small-cell lung cancer. Nat Commun 6, 6377 (2015).

715 21. Van Poznak, C. et al. Use of Biomarkers to Guide Decisions on Systemic Therapy for Women 716 With Metastatic Breast Cancer: American Society of Clinical Oncology Clinical Practice 717 Guideline. JCO 33, 2695-2704 (2015).

718 22. Bianchini, G., Balko, J. M., Mayer, I. A., Sanders, M. E. \& Gianni, L. Triple-negative breast 719 cancer: challenges and opportunities of a heterogeneous disease. Nat Rev Clin Oncol 13, $720 \quad 674-690(2016)$.

721 23. McAnena, P. F. et al. Breast cancer subtype discordance: impact on post-recurrence survival 722 and potential treatment options. BMC Cancer 18, 203 (2018).

723 24. Hulsbergen, A. F. C. et al. Subtype switching in breast cancer brain metastases: a 724 multicenter analysis. Neuro-Oncology 22, 1173-1181 (2020). 
725 25. Schrijver, W. A. M. E. et al. Receptor Conversion in Distant Breast Cancer Metastases: A

726 Systematic Review and Meta-analysis. JNCI: Journal of the National Cancer Institute 110,

$727 \quad 568-580(2018)$.

728 26. Lindström, L. S. et al. Clinically used breast cancer markers such as estrogen receptor,

729 progesterone receptor, and human epidermal growth factor receptor 2 are unstable

730 throughout tumor progression. Journal of clinical oncology : official journal of the American

$731 \quad$ Society of Clinical Oncology 30, 2601-8 (2012).

732 27. Aurilio, G. et al. A meta-analysis of oestrogen receptor, progesterone receptor and human

733 epidermal growth factor receptor 2 discordance between primary breast cancer and

734 metastases. European Journal of Cancer 50, 277-289 (2014).

735 28. Hoefnagel, L. D. C. et al. Receptor conversion in distant breast cancer metastases. Breast

$736 \quad$ cancer research : BCR 12, R75 (2010).

737 29. Shah, S. P. et al. The clonal and mutational evolution spectrum of primary triple-negative

738 breast cancers. Nature 486, 395-9 (2012).

739 30. Lindström, L. S. et al. Intratumor Heterogeneity of the Estrogen Receptor and the Long-term

740 Risk of Fatal Breast Cancer. JNCI: Journal of the National Cancer Institute 110, 726-733

741 (2018).

742 31. Ulz, P. et al. Inferring expressed genes by whole-genome sequencing of plasma DNA. Nature

$743 \quad$ Genetics 48, 1273-1278 (2016).

744 32. Snyder, M. W., Kircher, M., Hill, A. J., Daza, R. M. \& Shendure, J. Cell-free DNA Comprises an

745 in Vivo Nucleosome Footprint that Informs Its Tissues-Of-Origin. Cell 164, 57-68 (2016). 
33. Zhu, G. et al. Tissue-specific cell-free DNA degradation quantifies circulating tumor DNA burden. Nature Communications 12, 2229 (2021).

34. Sun, K. et al. Orientation-aware plasma cell-free DNA fragmentation analysis in open

$$
\text { chromatin regions informs tissue of origin. Genome research 29, 418-427 (2019). }
$$

35. Jiang, P. et al. Plasma DNA End-Motif Profiling as a Fragmentomic Marker in Cancer,

$$
\text { Pregnancy, and Transplantation. Cancer Discov 10, 664-673 (2020). }
$$

36. Lo, Y. M. D., Han, D. S. C., Jiang, P. \& Chiu, R. W. K. Epigenetics, fragmentomics, and

$$
\text { topology of cell-free DNA in liquid biopsies. Science 372, (2021). }
$$

754 37. Lai, B. et al. Principles of nucleosome organization revealed by single-cell micrococcal

755 nuclease sequencing. Nature 562, 281-285 (2018).

38. Cristiano, S. et al. Genome-wide cell-free DNA fragmentation in patients with cancer. Nature 570, 385-389 (2019).

39. Peneder, P. et al. Multimodal analysis of cell-free DNA whole-genome sequencing for

$$
\text { pediatric cancers with low mutational burden. Nat Commun 12, } 3230 \text { (2021). }
$$

40. Mouliere, F. et al. Enhanced detection of circulating tumor DNA by fragment size analysis.

$$
\text { Science Translational Medicine 10, eaat4921 (2018). }
$$

41. Underhill, H. R. et al. Fragment Length of Circulating Tumor DNA. PLOS Genet 12, 426-37

$$
\text { (2016). }
$$

42. Ulz, P. et al. Inference of transcription factor binding from cell-free DNA enables tumor

$$
\text { subtype prediction and early detection. Nature Communications 10, } 4666 \text { (2019). }
$$


44. Benjamini, Y. \& Speed, T. P. Summarizing and correcting the GC content bias in highthroughput sequencing. Nucleic Acids Research 40, e72-e72 (2012). gene transcription regulation - 2019 update. Nucleic Acids Research 47, D100-D105 (2019).

46. Albergaria, A. et al. Expression of FOXA1 and GATA-3 in breast cancer: the prognostic significance in hormone receptor-negative tumours. Breast Cancer Research 11, R40 (2009).

47. Corces, M. R. et al. The chromatin accessibility landscape of primary human cancers. Science 362, eaav1898 (2018).

48. Satpathy, A. T. et al. Massively parallel single-cell chromatin landscapes of human immune cell development and intratumoral T cell exhaustion. Nature Biotechnology 37, 925-936 (2019).

49. Weber, Z. T. et al. Modeling clonal structure over narrow time frames via circulating tumor DNA in metastatic breast cancer. Genome Medicine 13, 89 (2021).

50. Roth, A. et al. PyClone: statistical inference of clonal population structure in cancer. Nature methods 11, 396-8 (2014).

51. Wu, S. J. et al. Single-cell CUT\&Tag analysis of chromatin modifications in differentiation

52. Pierce, S. E., Granja, J. M. \& Greenleaf, W. J. High-throughput single-cell chromatin accessibility CRISPR screens enable unbiased identification of regulatory networks in cancer. Nat Commun 12, 2969 (2021). 
54. Wu, A. et al. Genome-wide plasma DNA methylation features of metastatic prostate cancer. J Clin Invest 130, 1991-2000 (2020).

55. Shen, S. Y. et al. Sensitive tumour detection and classification using plasma cell-free DNA methylomes. Nature 563, 579-583 (2018).

56. Liu, M. C. et al. Sensitive and specific multi-cancer detection and localization using methylation signatures in cell-free DNA. Annals of Oncology 31, 745-759 (2020).

57. Larson, M. H. et al. A comprehensive characterization of the cell-free transcriptome reveals tissue- and subtype-specific biomarkers for cancer detection. Nature Communications 12,

58. Kang, S. et al. CancerLocator: non-invasive cancer diagnosis and tissue-of-origin prediction using methylation profiles of cell-free DNA. Genome Biology 18, 53 (2017).

59. Chan, K. C. A. et al. Noninvasive detection of cancer-associated genome-wide hypomethylation and copy number aberrations by plasma DNA bisulfite sequencing. Proceedings of the National Academy of Sciences 110, 18761-18768 (2013).

60. Stover, D. G. et al. Association of Cell-Free DNA Tumor Fraction and Somatic Copy Number Alterations With Survival in Metastatic Triple-Negative Breast Cancer. JCO 36, 543-553 (2018).

61. Group (EBCTCG), E. B. C. T. C. Relevance of breast cancer hormone receptors and other factors to the efficacy of adjuvant tamoxifen: patient-level meta-analysis of randomised trials. The Lancet 378, 771-784 (2011). 
812 63. Slamon, D. J. et al. Human breast cancer: correlation of relapse and survival with

813 amplification of the HER-2/neu oncogene. Science 235, 177-182 (1987).

814 64. Curtis, C. et al. The genomic and transcriptomic architecture of 2,000 breast tumours

815 reveals novel subgroups. Nature 486, 346-352 (2012).

816 65. Nielsen, T. O. et al. A Comparison of PAM50 Intrinsic Subtyping with Immunohistochemistry

817 and Clinical Prognostic Factors in Tamoxifen-Treated Estrogen Receptor-Positive Breast

$818 \quad$ Cancer. Clinical Cancer Research 16, 5222-5232 (2010).

819 66. Karimzadeh, M., Ernst, C., Kundaje, A. \& Hoffman, M. M. Umap and Bismap: quantifying

820 genome and methylome mappability. Nucleic Acids Research 46, e120-e120 (2018).

821 67. Ramírez, F. et al. deepTools2: a next generation web server for deep-sequencing data

$822 \quad$ analysis. Nucleic Acids Research 44, W160-W165 (2016).

823 68. Jurka, J. Repbase Update: a database and an electronic journal of repetitive elements.

$824 \quad$ Trends in Genetics 16, 418-420 (2000).

825 69. Li, H. et al. The Sequence Alignment/Map format and SAMtools. Bioinformatics 25, 2078-

8262079 (2009).

827 70. Array programming with NumPy | Nature. https://www.nature.com/articles/s41586-020-

$828 \quad 2649-2$.

829 71. Picard Toolkit. (Broad Institute, 2021).

830 72. DePristo, M. A. et al. A framework for variation discovery and genotyping using nextgeneration DNA sequencing data. Nature Genetics 43, 491-498 (2011).

832 73. Li, H. Aligning sequence reads, clone sequences and assembly contigs with BWA-MEM. 00, 1-3 (2013). 
834 74. Danecek, P. et al. Twelve years of SAMtools and BCFtools. GigaScience 10, 1-4 (2021).

835 75. Weirauch, M. T. et al. Determination and Inference of Eukaryotic Transcription Factor

836 Sequence Specificity. Cell 158, 1431-1443 (2014).

837 76. Meuleman, W. et al. Index and biological spectrum of human DNase I hypersensitive sites.

$838 \quad$ Nature 584, 244-251 (2020).

839 77. Seabold, S. \& Perktold, J. Statsmodels: Econometric and Statistical Modeling with Python. in 92-96 (2010). doi:10.25080/Majora-92bf1922-011.

78. Dale, R. K., Pedersen, B. S. \& Quinlan, A. R. Pybedtools: a flexible Python library for manipulating genomic datasets and annotations. Bioinformatics 27, 3423-3424 (2011).

843 79. Quinlan, A. R. \& Hall, I. M. BEDTools: a flexible suite of utilities for comparing genomic features. Bioinformatics 26, 841-842 (2010).

$$
\text { Methods 17, 261-272 (2020). }
$$

847 81. Pedregosa, F. et al. Scikit-learn: Machine Learning in Python. Journal of Machine Learning

$$
\text { Research 12, 2825-2830 (2011). }
$$

849 82. Vallat, R. Pingouin: statistics in Python. Journal of Open Source Software 3, 1026 (2018).

\section{$851 \quad$ Acknowledgments}

852 We thank the many patients and their families for their generosity in contributing to this study. We 853 also thank Patricia Galipeau, Anat Zimmer and the Ha laboratory for helpful discussion and critical 854 reading of the manuscript. This work was supported by the National Institute of Health K22 855 CA237746 (to G.H.), the V Foundation Scholar Grant (to G.H.), Prostate Cancer Foundation 856 Young Investigator Award (to G.H.), the Fund for Innovation in Cancer Informatics Major Grant 
857 (to G.H.). This research was also supported by the NIH/NCI Cancer Center Support Grant P30

858 CA015704, Brotman Baty Institute for Precision Medicine, NIH (P50 CA097186; R01 CA2344715

859 to P.S.N; K08 CA252649 to H.A.P.; P50 CA168504 to H.A.P.; K12 CA076930 to J.H.; T32

860 HL007093 to J.H.), CDMRP W81XWH-18-10406 (to P.S.N), Komen Breast Cancer Foundation

861 Catalyst Research Grant (to H.A.P.). Scientific Computing Infrastructure was funded by ORIP

862 Grant S10OD028685.

863

864 Author contributions

865 A-L.D. and G.H. conceived the study, designed all the experiments, and wrote the manuscript. A-

866 L.D. developed and implemented the Griffin method and performed all the analysis. M.K., H.L.,

867 A.E.C, C.K., A.C.H.H., K.C. contributed to the analysis. K.S., H.A.P, D.G.S. provided clinical data.

868 Z.T.W. provided clonality results. J.H., R.D.P., N.D.S., M.A., J.R. contributed to analysis

869 discussions. P.P., V.A.A., P.S.N., H.A.P., D.G.S., D.M. contributed to discussions, provided

870 guidance and interpretation of results. G.H. supervised the study. All authors reviewed and edited

871 the manuscript.

872

\section{$873 \quad$ Competing interests}

874 The authors have filed a pending patent application on methodologies developed in this 875 manuscript (A-L.D., G.H.). All other authors declare no competing interests. 
medRxiv preprint doi: https://doi.org/10.1101/2021.08.31.21262867; this version posted September 3, 2021. The copyright holder for this preprint (which was not certified by peer review) is the author/funder, who has granted medRxiv a license to display the preprint in perpetuity.

It is made available under a CC-BY-NC-ND 4.0 International license .

a

Accessible sites or actively bound TFBSs

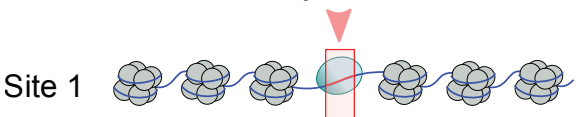

Site 2 \&

Site 3 \&2 82 \&
Inaccessible sites or non-actively bound TFBSs

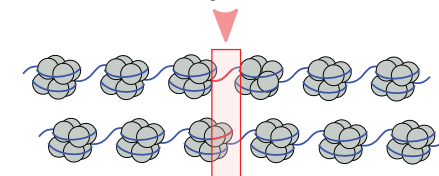

\&2 82,828

cfDNA fragments
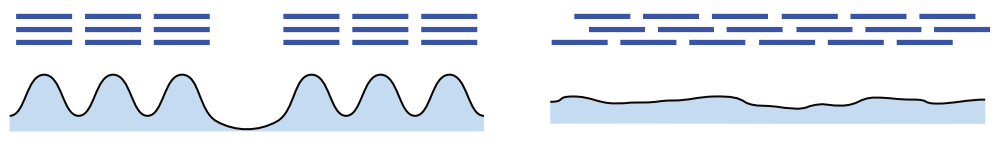

Coverage

b

\section{Cancer patient plasma cell-free DNA}

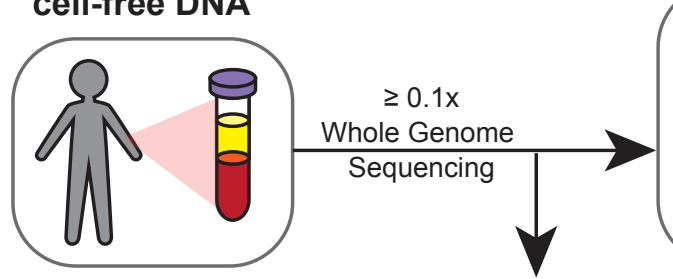

2. Compute GC-corrected fragment midpoint coverage

Sites of interest

Cancer \& subtype specific chromatin accessibility Transcription factor binding sites
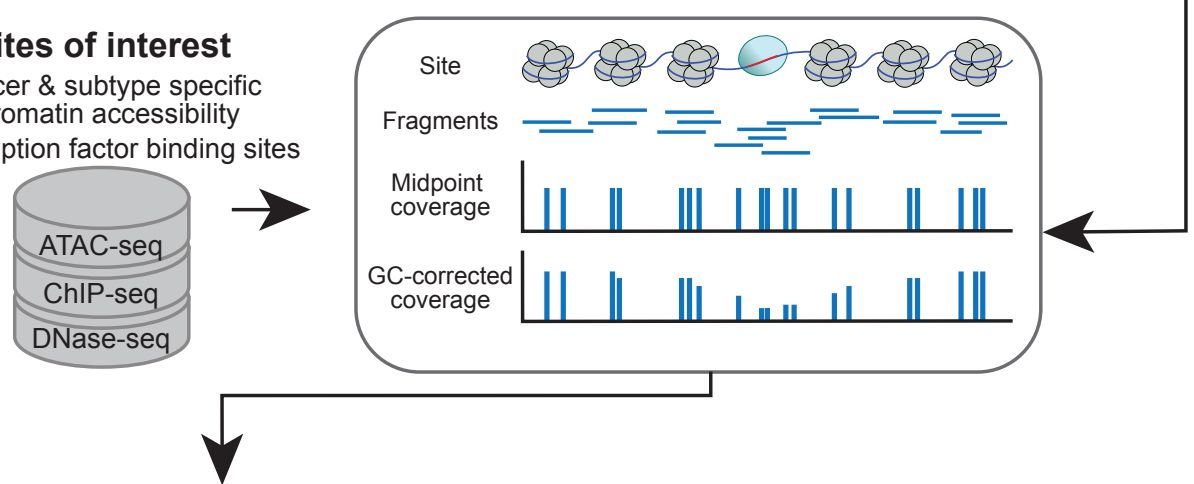

3. Average all sites in a group (e.g. tissue, subtype, transcription factor)

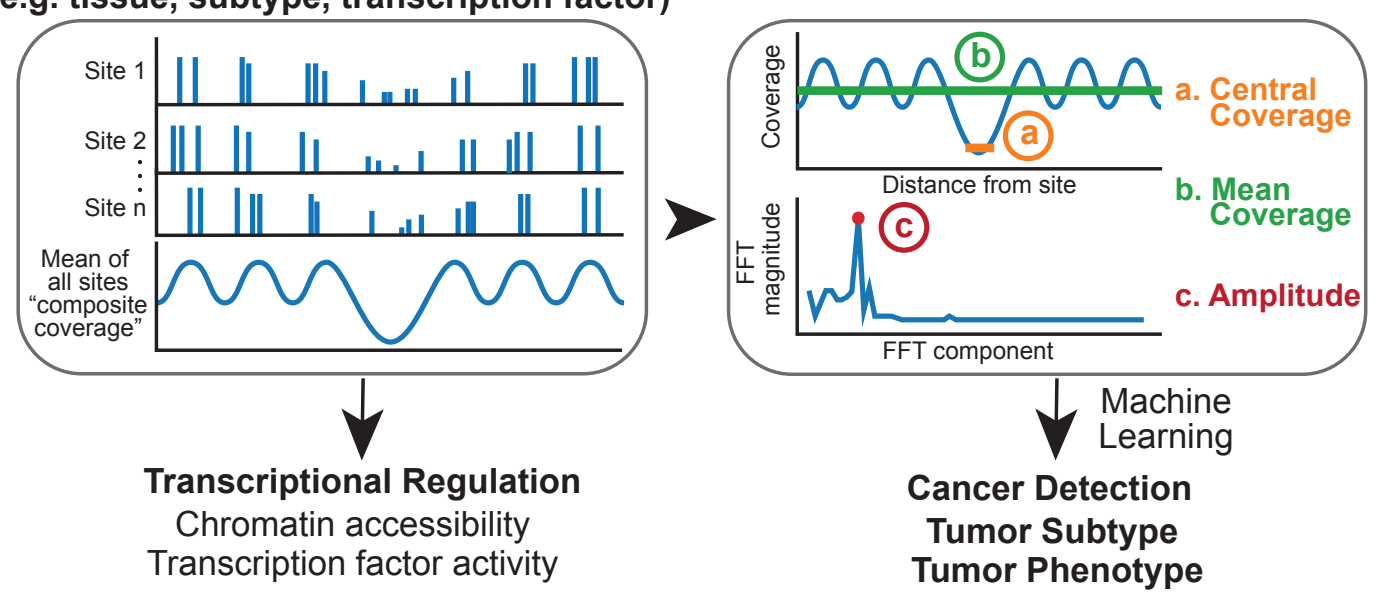




\section{$876 \quad$ Figure legends}

877 Fig. 1 Griffin framework for cfDNA nucleosome profiling to predict cancer subtypes and

878 tumor phenotype. (a) Illustration of a group of accessible sites (left panel) and inaccessible sites

879 (right panel), such as a TFBS. The nucleosomes (in grey) are positioned in an organized manner 880 around the accessible sites (red box; left panel), but not around the inaccessible ones (right 881 panel). These nucleosomes protect the DNA from degradation when it is released into peripheral 882 blood. The protected fragments from the plasma are sequenced and aligned, leading to a 883 coverage profile which reflects the nucleosome protection in the cells of origin. (b) Griffin workflow 884 for cfDNA nucleosome profiling analysis. cfDNA whole genome sequencing (WGS) data with $\geq$ $8850.1 \times$ coverage is aligned to hg38 genome build. (1) For each sample, fragment-based GC bias is 886 computed for each fragment size. (2) Sites of interest are selected from any assay. Paired-end 887 reads aligned to each site are collected, fragment midpoint coverage is counted, and corrected 888 for GC bias to produce a coverage profile. (3) Coverage profiles from all sites in a group (e.g., 889 open chromatin for tumor subtype) are averaged to produce a composite coverage profile. 890 Composite profiles are normalized using the surrounding region $(-5 \mathrm{~kb}$ to $+5 \mathrm{~kb})$. (4) Three 891 features are extracted from the composite coverage profile: central coverage (coverage from -30

$892 \mathrm{bp}$ to $+30 \mathrm{bp}$ from the site; orange ' $a$ '), mean coverage (between $-1 \mathrm{~kb}$ to $+1 \mathrm{~kb}$; green ' $\mathrm{b}$ '), and 893 amplitude calculated using a Fast-Fourier Transform (FFT) (red 'c'). 
a

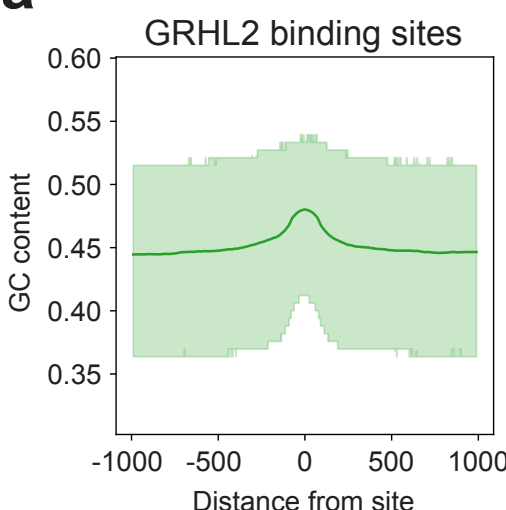

b

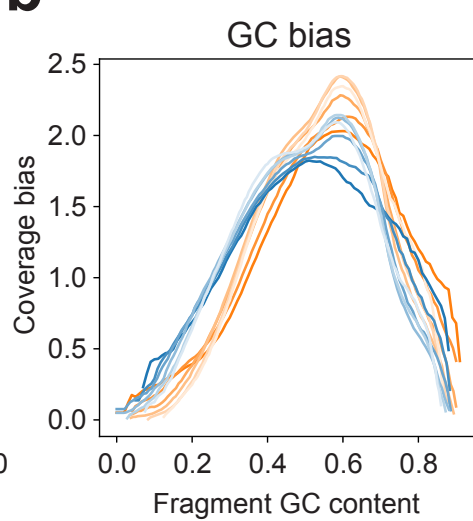

C

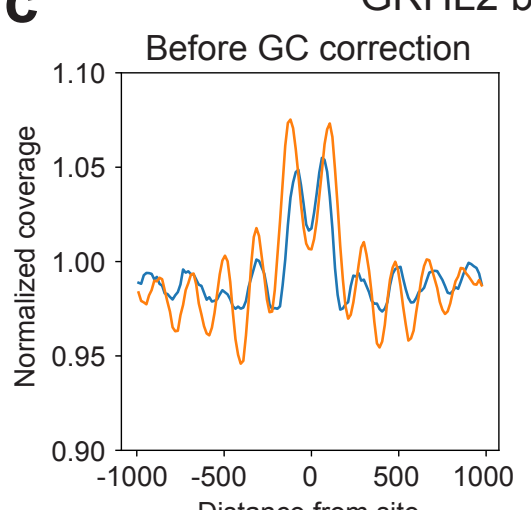

Distance from site d
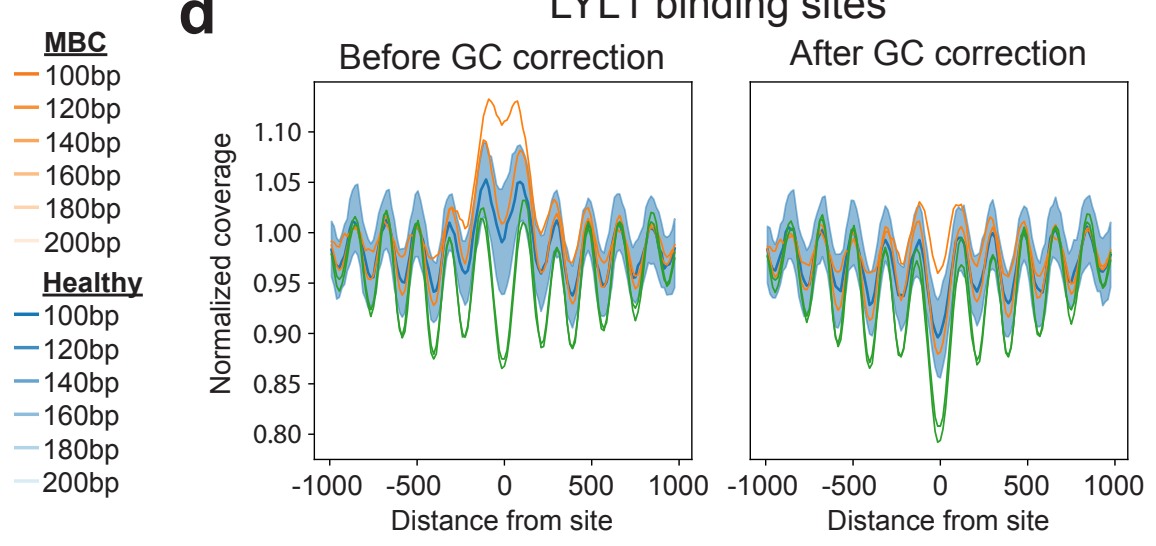

${ }_{n=191}^{\operatorname{MBC}(0.1-0.3 \mathrm{x})}-{ }_{\mathrm{n}=2}^{\mathrm{MBC}(9-25 \mathrm{x})}-{ }_{\mathrm{n}=2}^{\text {Healthy }}(17-20 \mathrm{x})$

e

LYL1 binding sites
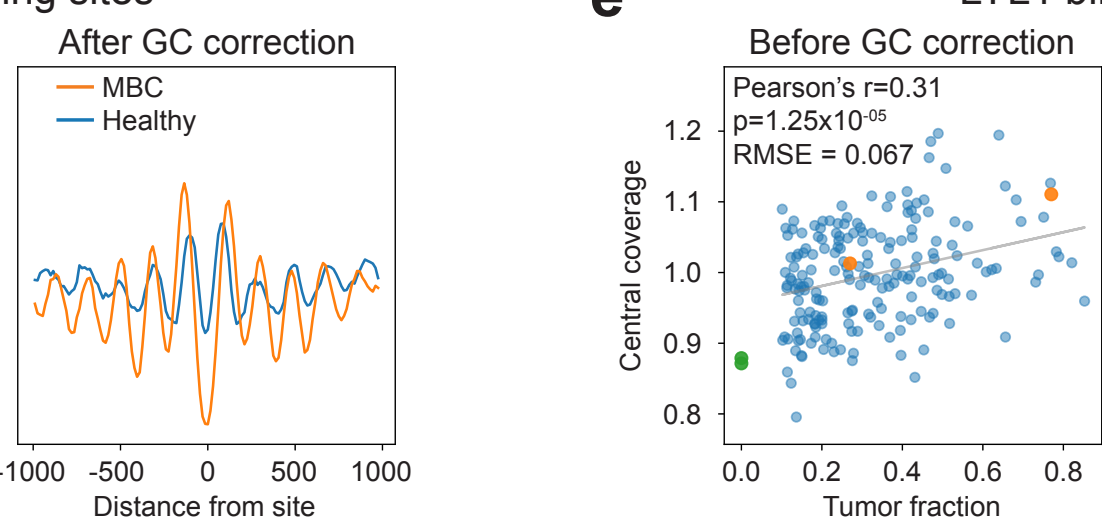

\section{After GC correction}

Pearson's $r=0.63$
$p=2.03 \times 10^{-22}$

RMSE $=0.041$

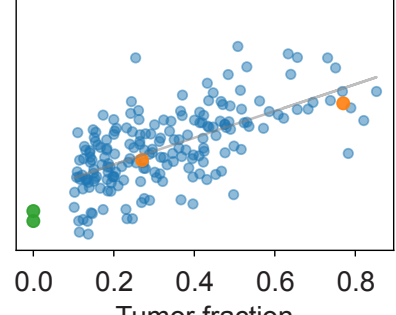

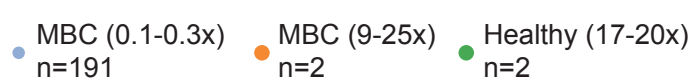

f

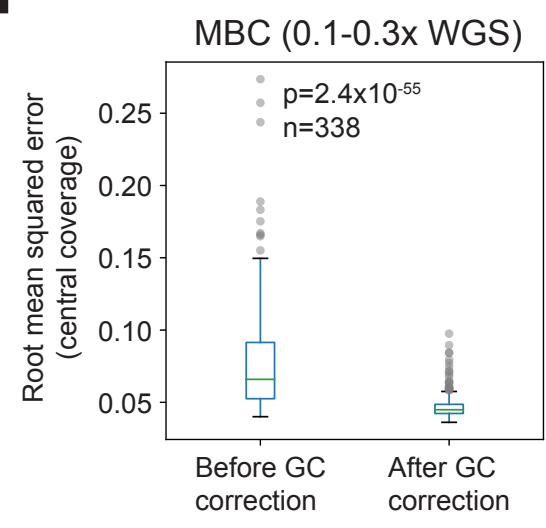

g

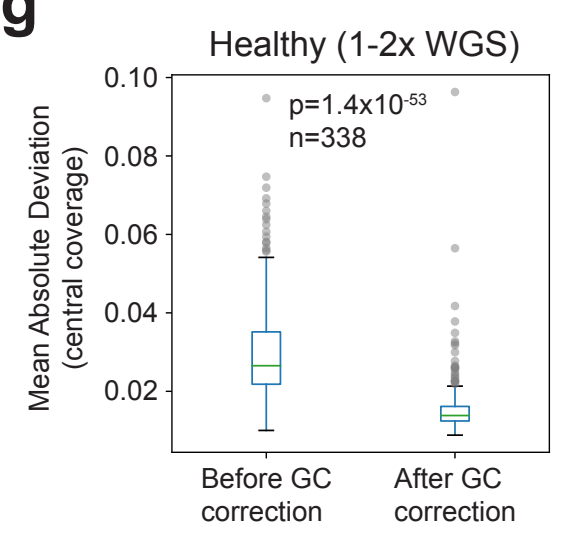



cfDNA. (a) Aggregated GC content at 10,000 GRHL2 binding sites and its surrounding 2kb region. Mean GC content (line) and interquartile range (green shading) are shown. (b) cfDNA GC bias is unique to each sample and each fragment length. GC bias computed for cfDNA from a healthy donor (HD_46; blue shades) and a metastatic breast cancer (MBC_315; orange shades) sample are shown for various fragment sizes. (c) Composite coverage profile of 10,000 GRHL2 binding sites before and after GC correction, shown for HD_46 (blue) and MBC_315 (orange). Before GC correction, the 'central coverage' has a higher value due to effects of GC bias which can obscure differential signals between samples. After GC correction, the central coverage of the MBC sample has lower value, which is consistent with increased GRHL2 activity in breast cancer but not immune cells making up the healthy donor sample. (d) Composite coverage profiles of 10,000 LYL1 sites before and after GC correction, shown for two MBC samples with deep WGS (9-25x, orange), two healthy donors (17-20x, green), and 191 MBC samples with ULPWGS (0.1-0.3x, blue). Median +/- IQR of 191 ULP-WGS samples is shown with blue shading. Lower 'central coverage' corresponding to greater site accessibility in the healthy donor samples is expected because LYL1 is a transcription factor associated with hematopoiesis. (e) cfDNA

911 tumor fraction and central coverage correlation for LYL1, shown for ULP-WGS (0.1-0.3x, n=191)

912 and WGS $(9-25 x, n=2)$ of MBC and healthy donors $(17-20 x, n=2)$ samples. cfDNA contains a 913 mixture of tumor and blood cells; therefore, central coverage value is expected to be positively 914 correlated with tumor fraction (lower represents increased accessibility). After GC correction, the 915 correlation (for the MBC ULP-WGS samples) is much stronger based on Pearson's $r$ correlation 916 coefficient. Root mean squared error (RMSE) of the linear fit is shown. (f) Boxplots showing the 917 distribution of the RMSE (linear fit between central coverage and tumor fraction in the MBC ULP918 WGS dataset [0.1-0.3x, n=191]) across the 338 TFs, before and after GC correction. The boxed 919 range represents the median \pm IQR, whiskers represent the range of the non-outlier data 920 (maximum extent is $1.5 x$ the IQR). Outliers are plotted in grey. $p$-value was calculated using the 
medRxiv preprint doi: https://doi.org/10.1101/2021.08.31.21262867; this version posted September 3, 2021. The copyright holder for this preprint

(which was not certified by peer review) is the author/funder, who has granted medRxiv a license to display the preprint in perpetuity. It is made available under a CC-BY-NC-ND 4.0 International license .

921 Wilcoxon signed-rank test (two-sided). (g) Boxplots showing the distribution of the mean absolute

922 deviation (of the central coverage across 215 healthy donors [1-2x WGS]) across the 338 TFs,

923 before and after GC correction. Box elements are the same as (f). p-value was calculated using

924 the Wilcoxon signed-rank test (two-sided).

925 
a

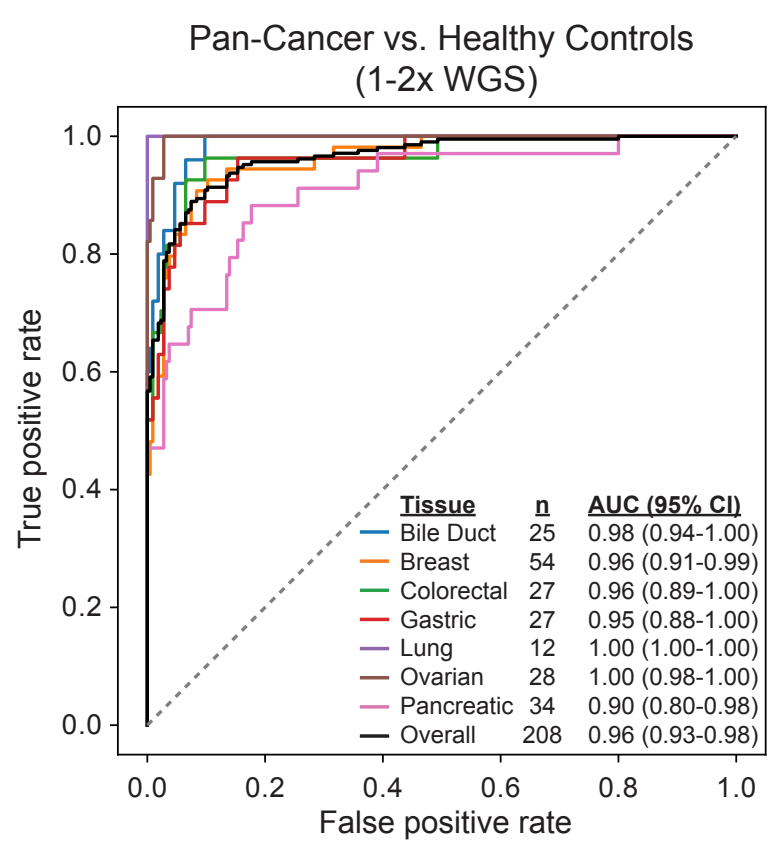

C

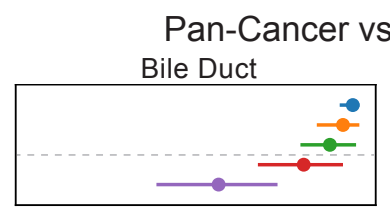

Breast

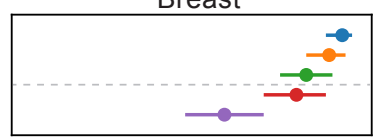

Colorectal

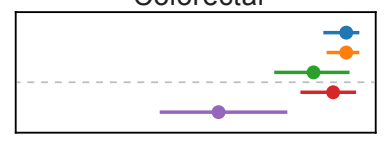

Gastric

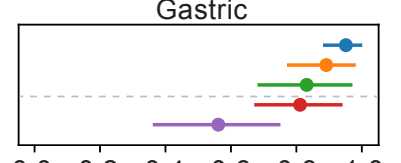

$\begin{array}{lllllllllllll}0.0 & 0.2 & 0.4 & 0.6 & 0.8 & 1.0 & 0.0 & 0.2 & 0.4 & 0.6 & 0.8 & 1.0\end{array}$

AUC b

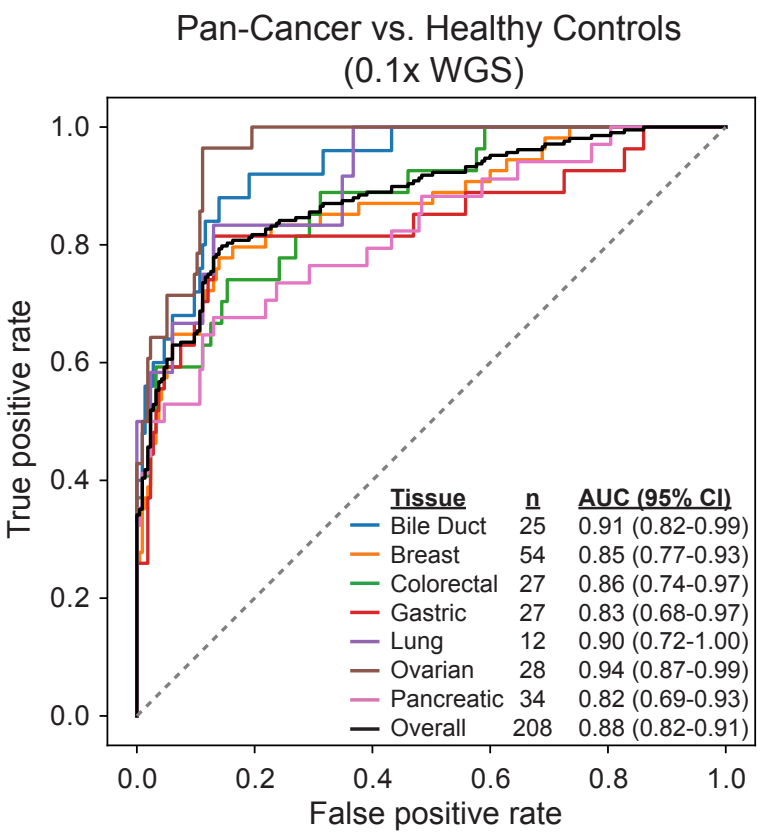

d

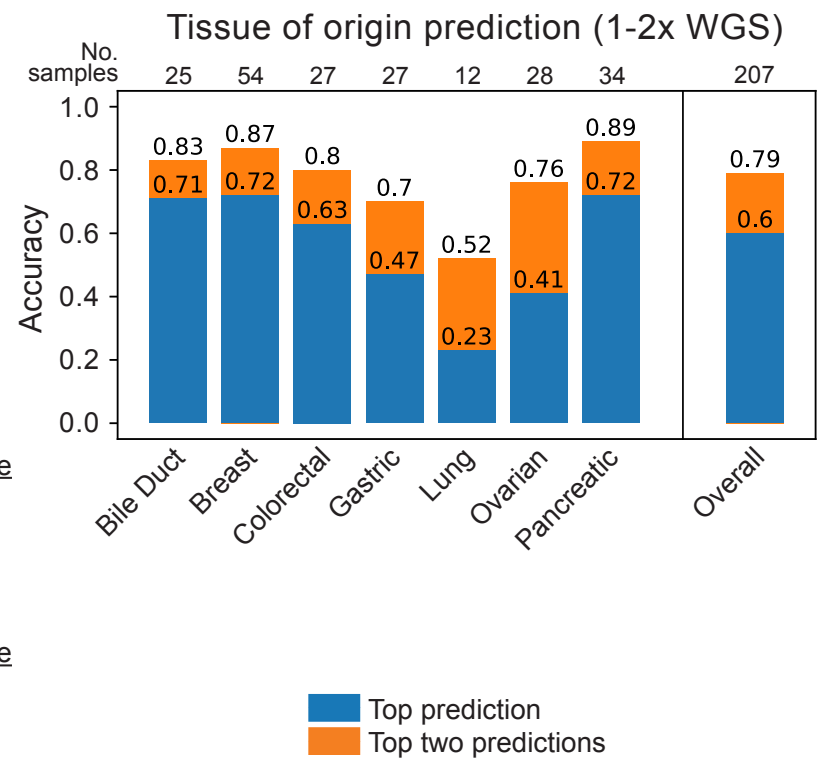


medRxiv preprint doi: https://doi.org/10.1101/2021.08.31.21262867; this version posted September 3, 2021. The copyright holder for this preprint (which was not certified by peer review) is the author/funder, who has granted medRxiv a license to display the preprint in perpetuity.

It is made available under a CC-BY-NC-ND 4.0 International license.

926 Fig. 3 Griffin enables accurate cancer detection and tissue-of-origin prediction. (a) Receiver

927 operator characteristic (ROC) curve for logistic regression classification of cancer $(n=208)$ vs.

928 healthy controls $(n=215)^{38}$ using nucleosome profiles around TFBSs in 1-2x WGS data. ROC for

929 each cancer type vs. healthy are shown. 95\% confidence intervals (Cls) were obtained by

930 bootstrapping. Duodenal cancer $(n=1)$ is not shown. (b) ROC for logistic regression classification

931 of cancer using the same TFBSs feature set applied to the same dataset downsampled to $0.1 \mathrm{x}$

932 WGS coverage. (c) Area under the ROC curve (AUC) values for logistic regression models using

933 different feature sets collected from nucleosome profiling around TFBSs. The fragment size

934 range, sample coverage, and nucleosome profiling tool (Griffin and Ulz pipelines) are indicated.

$93595 \%$ Cls were obtained by bootstrapping. (d) Accuracy of a multinomial logistic regression model

936 used to predict tissue-of-origin in 207 cancer patients (duodenal cancer was excluded). The

937 accuracy of the top prediction and top two predictions by the model are shown for each individual

938 cancer type and overall, for all cancer types combined. 
medRxiv preprint doi: https://doi.org/10.1101/2021.08.31.21262867; this version posted September 3, 2021. The copyright holder for this preprint (which was not certified by peer review) is the author/funder, who has granted medRxiv a license to display the preprint in perpetuity. It is made available under a CC-BY-NC-ND 4.0 International license .

a

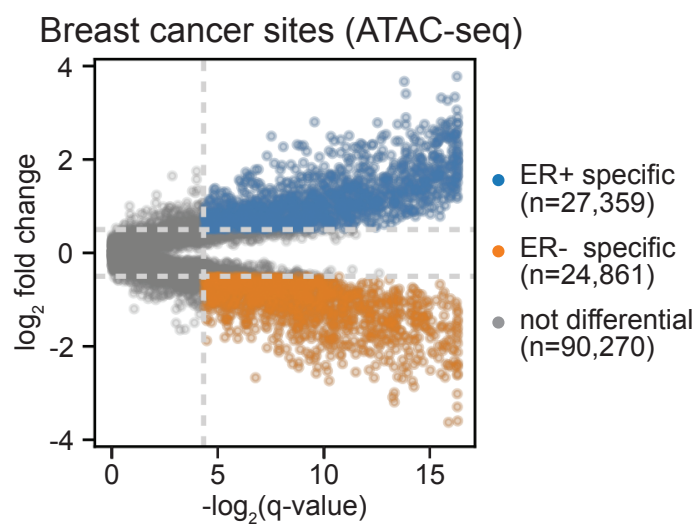

b

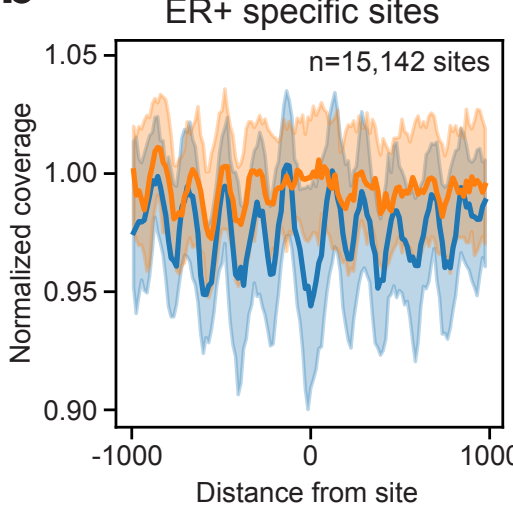

MBC ULP-WGS $(0.1-0.3 \mathrm{x})$ cfDNA

E- Clinical ER+ (99 samples)

- Clinical ER- (92 samples)

C

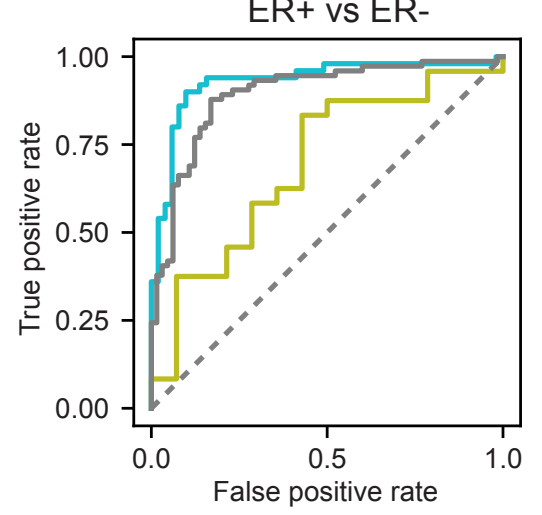

$\underline{\text { TFx }} \underline{\mathrm{n}}$ Accuracy $\quad \underline{\mathrm{AUC}}$

$-\quad \overline{0.05}-0.1 \quad 38 \quad \frac{0.64(0.38-0.89)}{38} \quad 0.68(0.34-0.96)$

$\begin{array}{lllll}-\geq 0.1 & 101 & 0.88(0.78-0.97) & 0.93 & (0.85-0.99)\end{array}$

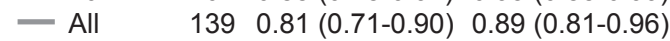

d

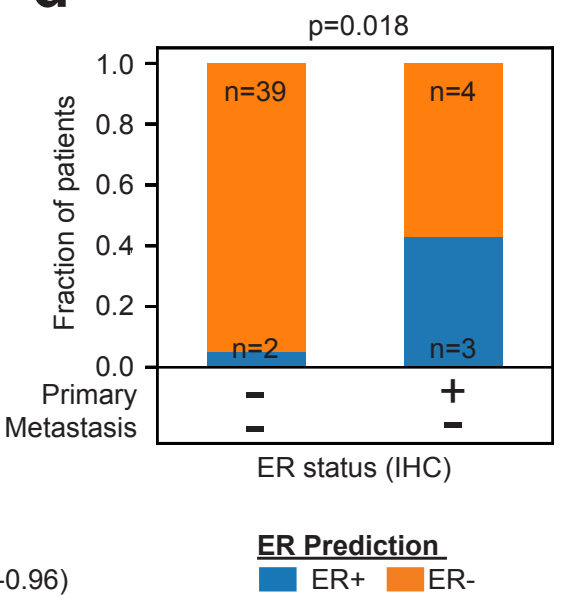

f

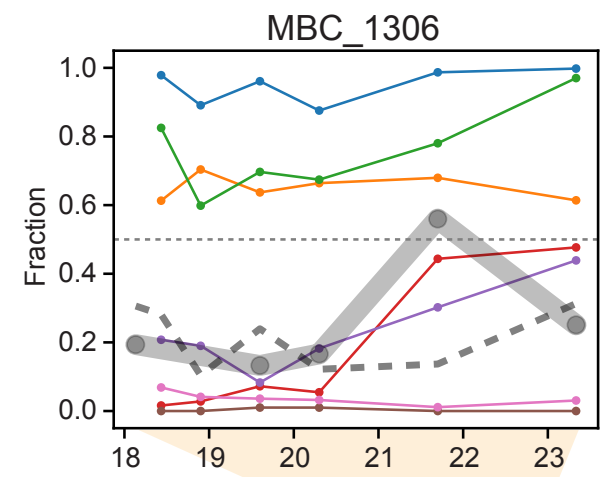

ER- ER-

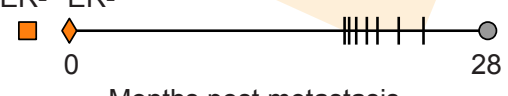

Months post metastasis

Probability of ER+

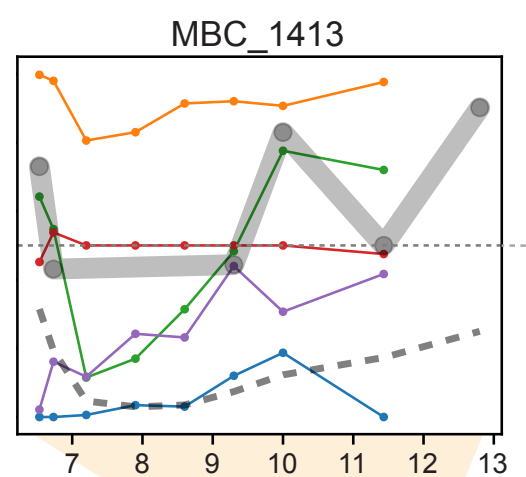

$E R+E R-$
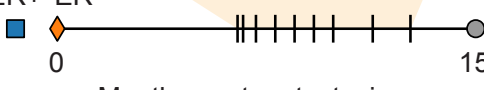

Months post metastasis

Clonal Cluster Cellular Prevalence

$\rightarrow 1 \rightarrow 2 \rightarrow 3 \rightarrow 4$

$\rightarrow 5 \rightarrow 6 \rightarrow 7$
ER- specific sites

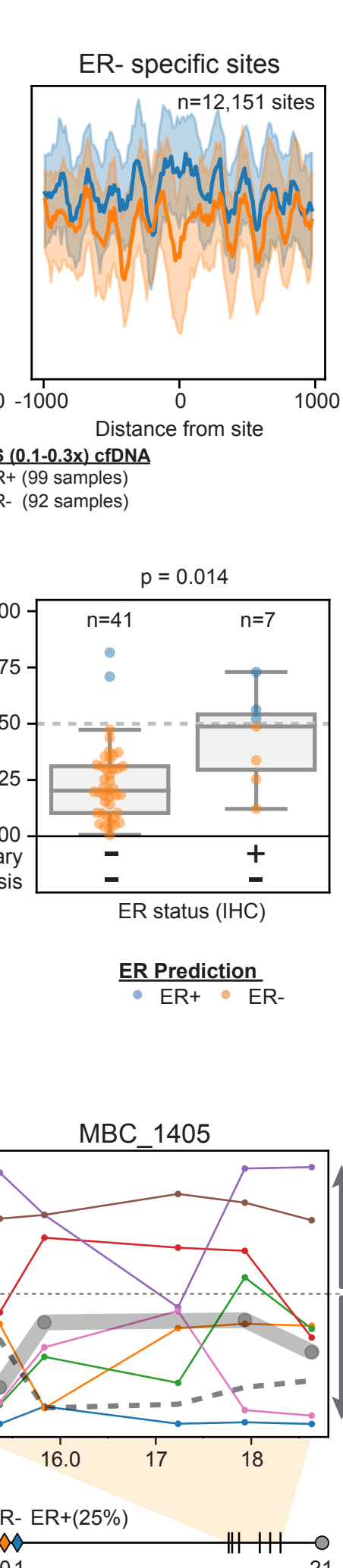

e

ER Prediction

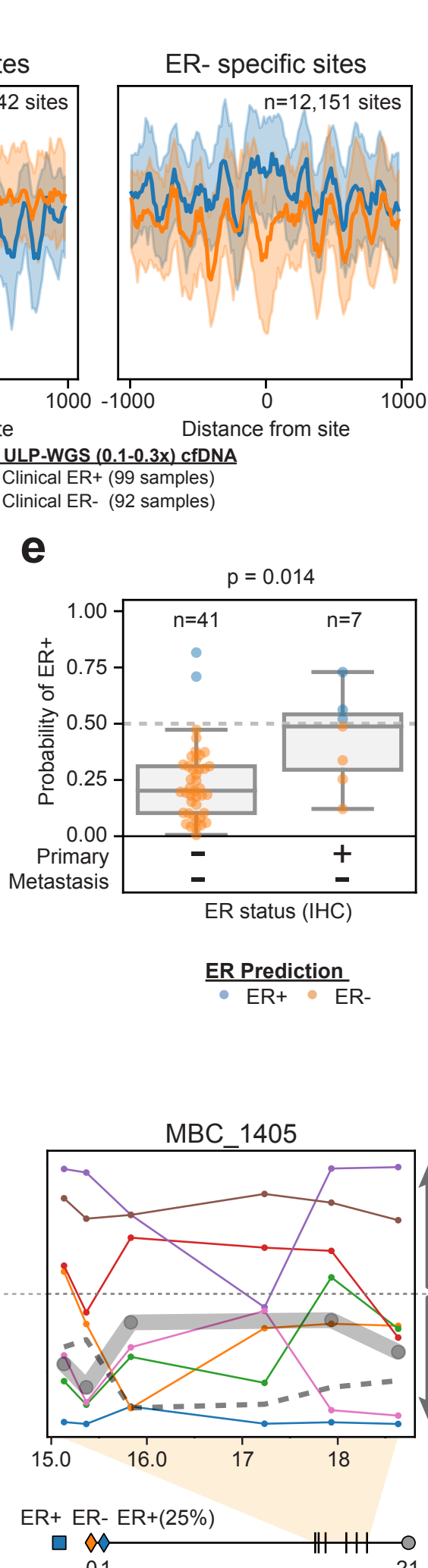

MBC_1405

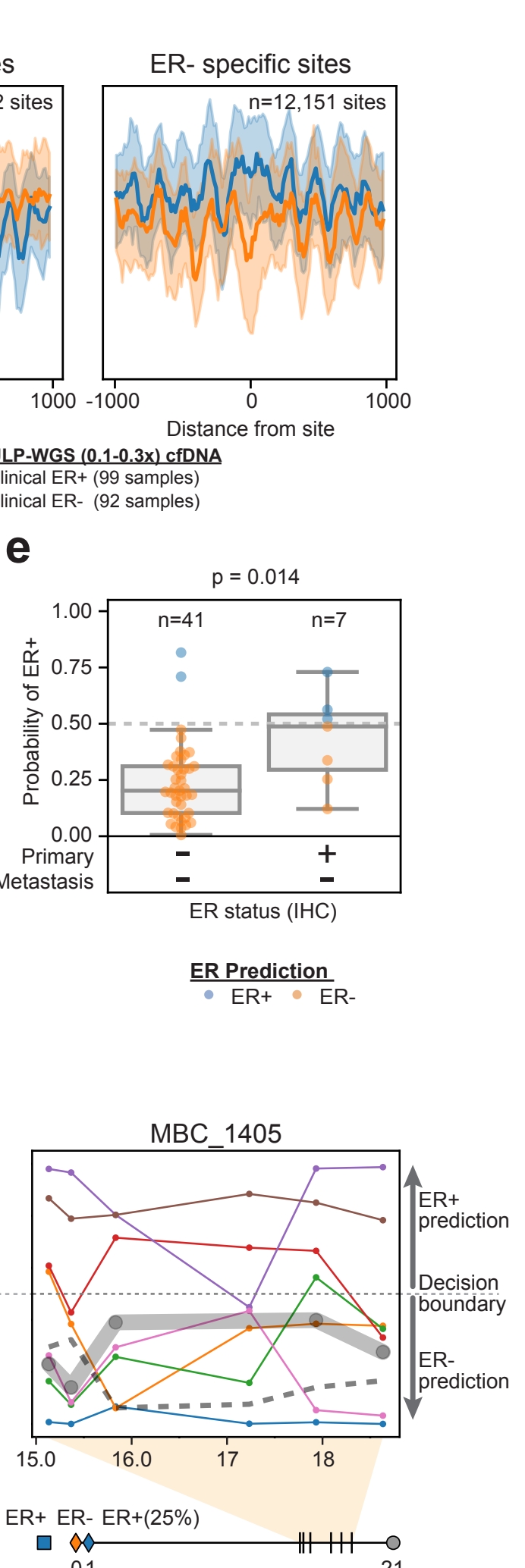

$E R+E R-E R+(25 \%)$

01

Months post metastasis

Timeline

$\square$ Primary I cfDNA sample

$\diamond$ Metastasis O Death 
941 ultra-low pass WGS. (a) ER+ and ER- specific open chromatin sites were selected from assay

942 for transposase-accessible chromatin using sequencing (ATAC-seq) data from ER+ $(n=44)$ and 943 ER- $(n=15)$ breast tumors in The Cancer Genome Atlas (TCGA). ${ }^{47}$ Sites were selected using a 944 Mann-Whitney-U (two-sided) test with Benjamini-Hochberg p-value adjustment (q-value) for each 945 site, and the $\log _{2}$ fold change was also calculated. Sites with a q-value $<0.05$ and a $\log _{2}$ fold 946 change of $>0.5$ or $<-0.5$ were considered differential. (b) Composite coverage profiles (median \pm 947 IQR) for ER+ specific $(n=15,142)$ and ER- specific $(n=12,151)$ sites are shown for MBC patients 948 ( $\geq 0.1$ tumor fraction) separated by clinical ER status (ER+, n=99; ER-, $n=92)$. Sites shared with hematopoietic cells were excluded. ${ }^{48}$ (c) Receiver operator characteristic (ROC) curve for a logistic regression model predicting ER+ and ER- subtype. ROC curve, accuracy and AUC are

951 shown for all patients and for patients grouped by tumor fraction (TFx), 0.05-0.1 and $\geq 0.1$. 95\%

952 Cls were obtained by bootstrapping. For patients with multiple samples, the first sample with 953 tumor fraction $>0.05$ was used. (d) Subtype prediction in patients with metastatic ER- breast 954 cancer separated by clinical primary tumor ER status. P-value was calculated using a Fisher's 955 exact test (two-sided). (e) Boxplot showing the distribution of probabilities of ER+ for the same 956 patients as in (d). The boxed range represents the median $\pm I Q R$, whiskers represent the range 957 of the non-outlier data (maximum extent is $1.5 x$ the IQR). All individual points are plotted. P-value 958 calculated using ANCOVA with tumor fraction as a covariate. (f) Cellular prevalence of clonal 959 clusters, ER+ prediction probability (grey line), and tumor fraction (dashed line) for multiple 960 plasma samples shown for patients, MBC 1306, MBC 1413, and MBC 1405. Cellular prevalence 961 was obtained from a previous study using PyClone analysis of whole exome and targeted panel 962 sequencing of the same samples; analysis was performed independently for each patient. ${ }^{49}$ 963 Decision boundary for ER+ $(\geq 0.5)$ and ER- $(<0.5)$ is indicated with dotted line. Timelines in months 964 from metastatic diagnosis to death are shown for each patient. For patient MBC_1405, two 
medRxiv preprint doi: https://doi.org/10.1101/2021.08.31.21262867; this version posted September 3, 2021. The copyright holder for this preprint

(which was not certified by peer review) is the author/funder, who has granted medRxiv a license to display the preprint in perpetuity.

It is made available under a CC-BY-NC-ND 4.0 International license.

966 biopsy taken at metastatic diagnosis), and one was moderately ER+ (25\% ER staining, bone

967 lesion, taken 26 days after diagnosis). This patent was considered ER+ for the purpose of the

968 classifier (see Methods) but predicted as ER- for all timepoints.

969 